Preprint typeset in JHEP style. - PAPER VERSION

\title{
Graded D-branes and skew categories
}

\author{
C. I. Lazaroiu \\ Trinity College Dublin \\ Dublin 2 \\ Ireland \\ calin@maths.tcd.ie
}

\begin{abstract}
I describe extended gradings of open topological field theories in two dimensions in terms of skew categories, proving a result which alows one to translate between the formalism of graded open $2 \mathrm{~d}$ TFTs and equivariant cyclic categories. As an application of this formalism, I describe the open 2d TFT of graded D-branes in Landau-Ginzburg models in terms of an equivariant cyclic structure on the triangulated category of 'graded matrix factorizations' introduced by Orlov. This leads to a specific conjecture for the Serre functor on the latter, which generalizes results known from the minimal and Calabi-Yau cases. I also give a description of the open 2d TFT of such models which manifestly displays full grading induced by the vector-axial R-symmetry group.
\end{abstract}




\section{Contents}

1. Graded open topological field theories in two dimensions 4

2. Graded open 2d TFTs with shifts $\quad 6$

2.1 Mathematical preparations 6

2.2 G-graded open 2d TFTs with shifts $\quad 10$

3. $\quad G$-graded dG categories $\quad 13$

3.1 Integrable modules over a curved differential graded algebra 15

4. Application to graded B-type branes in Landau-Ginzburg models 21

4.1 The dG category of graded D-branes 21

4.2 Shift functor and distinguished twist functor 23

4.3 Traces 24

4.4 The graded topological field theory with shifts 25

$\begin{array}{ll}4.5 & \text { Component description }\end{array}$

$\begin{array}{lr}\text { 5. Conclusion } & 28\end{array}$

A. Equivariant Serre functors. $\quad 29$

B. Worldsheet analysis of R-symmetries in LG models 30 


\section{Introduction}

In open topological string models, the space of boundary or boundary condition changing observables is generically $\mathbb{Z}_{2}$-graded. In certain cases this grading extends to a larger Abelian group $G$, such as the group of characters of the surviving vector and axial R-symmetry of the worldsheet model. In such theories, the category $\mathcal{G}$ of boundary sectors is $G$-graded, and the general formalism of two-dimensional open topological field theories $[1,2]$ must be enriched to account for this supplementary structure.

A basic technical device in the study of open $2 \mathrm{~d}$ TFTs is the idea of reduction to an ungraded category, which is implicit in most studies of homological mirror symmetry. This reduces the structure of $[1,2]$ to a subset of the usual theory of Serre functors [3]. Such a reduction is possible provided that the graded category of boundary sectors admits sufficient symmetries which allow one to trade in the grading for an action on the collection of objects. In the generic, $\mathbb{Z}_{2}$-graded case, this translates the structure of [1] into a Calabi-Yau category whose shift functor squares to the identity. A similar relation exists when the open $2 \mathrm{~d}$ TFT is $\mathbb{Z}_{\omega}$-graded, the corresponding object being a Calabi-Yau category whose shift functor has order $\omega$. Finally, a $\mathbb{Z}$-graded open 2d TFT corresponds to a Calabi-Yau category whose shift functor generates a $\mathbb{Z}$-subgroup of the automorphism group.

When working with extended gradings by larger Abelian groups $G$, this point of view leads to the theory of categories endowed with a group action, and their skew categories [4]. In this paper, I discuss the general framework allowing for such a reduction, giving a precise relation between the physics description in terms of a $G$-graded category endowed with natural and nondegenerate traces and a more familiar mathematical description in terms of 'equivariant cyclic categories', i.e. ungraded categories endowed with an (Abelian) group action and a special type of Serre functor. This leads to a reconstruction result which allows one to recover the physics description from more traditional mathematical data. When the group $G$ is sufficiently large, this result shows how the formalism of $[1,2]$ can produce non-Calabi-Yau categories. As an application, I give a complete treatment of grading issues for the case of topological Landau-Ginzburg models, refining previous discussion of such theories.

A common example of extended grading in open 2d TFTs arises from open sigma models which admit an unbroken subgroup $\Gamma$ of vector and axial R-symmetries. In this situation, the category $\mathcal{G}$ of boundary sectors admits a grading by the Abelian group of characters $G=\left(\Gamma / \Gamma_{0}\right)^{*}$, where $\Gamma_{0}$ is the trivially acting subgroup. The main application considered in this paper concerns B-type topological Landau-Ginzburg models with target $\mathbb{C}^{n}$ and bulk superpotential $W$, whose D-branes were constructed in $[5,6,7,8,9]$. Using the normalization in which vector R-charges are integral, the bulk 
superpotential preserves the full worldsheet $U(1)_{V}$ R-symmetry provided that it is a homogeneous polynomial of the bulk superfields, whose degree we denote by $h=\operatorname{deg} W$. The model also admits an axial R-symmetry, which is broken to a $\mathbb{Z}_{2}$ subgroup by the bulk superpotential. This is responsible for the appearance of supermodules in the description of $[5,6,7,8]$ and gives an unbroken total R-symmetry group $\Gamma=$ $U(1) \times \mathbb{Z}_{2}$. It turns out that the grading group $G$ depends on the parity of $h$. In fact, direct analysis of the worldsheet shows that the trivially acting subgroup $\Gamma_{0}$ is trivial for even $h$ and isomorphic to $\mathbb{Z}_{2}$ for odd $h$. As a consequence, $G$ equals $\mathbb{Z} \times \mathbb{Z}_{2}$ or $\mathbb{Z}$, depending on the parity of $h$. Taking this fact into account, one finds that the category of 'graded D-branes' (i.e. those B-type branes which preserve the full vector Rsymmetry) is most conveniently described as the category of finitely generated $G$-graded integrable modules over a curved differential graded algebra with trivial differential. This simplifies the more traditional description in terms of matrix factorizations, and allows one to give a complete construction of the differential graded category underlying graded D-branes, in a formulation which explicitly displays the worldsheet symmetry of the model. The formulation used in this paper is a Landau-Ginzburg extension of the approach through graded superconnections of total degree one used in $[13,14,15$, 16, 17, 18, 19, 20] for B-type branes on Calabi-Yau manifolds.

Using this description, one finds that the resulting open $2 \mathrm{~d}$ TFT is described by a ' $G$-graded category with shifts', whose shift functors are compatible with the invariant traces. Restricting to morphisms of trivial degree gives an equivalent description in terms of a $G$-equivariant cyclic structure on the triangulated category of graded matrix factorizations constructed in [10]. In particular, we find that the traces of [5] lead to a precise conjecture for a Serre functor on this triangulated category. Finally, the correspondence result mentioned above allows one to recover the full open $2 \mathrm{~d}$ TFT from this triangulated category, provided that the latter is considered together with this Serre functor.

The paper is organized as follows. In Section 1, we discuss the mathematical notion of open 2d TFTs with extended grading, and explain how examples of such gradings arise from worldsheet R-symmetries. In Section 2, we discuss the special case of 'graded open 2d TFTs with shifts', which allows for an equivalent description in terms of ungraded categories endowed with an equivariant cyclic structure. After developing some mathematical machinery, we give a result which translates between the two descriptions. This gives a precise relation between the nondegenerate traces required by the physics formalism and the usual theory of Serre functors [3]. Section 3 discusses dG categories with extended grading, focusing on the example of integrable modules with extended grading over a curved dGA, which is relevant for Landau-Ginzburg models. In Section 4, we apply this machinery to the case of Landau-Ginzburg models with 
target $\mathbb{C}^{n}$. Using the worldsheet analysis Appendix B, we show that graded matrix factorizations can be described as the $G$-graded dG category of integrable modules over a curved differential graded algebra, whose total cohomology category recovers the desired $G$-graded open 2d TFT with shifts. After giving the precise homogeneity property of the traces, we use the general correspondence of Section 2 to extract a conjecture for the Serre functor on the triangulated category of matrix factorizations, and check it against known results in the Calabi-Yau and minimal model case $[11,12]$. This fills in a gap in the relation between the mathematical analysis of [10] and the physical description of open 2d TFTs. Appendix A contains a general discussion of equivariant Serre functors, while Appendix B gives the worldsheet analysis which leads to the description used in Section 4.

\section{Graded open topological field theories in two dimensions}

It was shown in $[1,2]$ that the boundary sector of a topological field theory in two dimensions is encoded by category-theoretic data, which can be viewed as an abstract definition of the notion of open $2 \mathrm{~d}$ topological field theory (TFT). As shown in loc. cit., such a theory is described by a Hom-finite ${ }^{1} \mathbb{C}$-category $\mathcal{G}$, whose objects are the boundary sectors, and whose morphisms are the boundary/boundary condition changing observables; the morphism compositions correspond to the product of such observables. A distinguishing feature of such theories is the existence of nondegenerate invariant linear maps $\operatorname{tr}_{a}: \operatorname{Hom}_{\mathcal{G}}(a, a) \rightarrow \mathbb{C}$, which arise from the two-point functions on the disk. In the generic case, the category $\mathcal{G}$ is $\mathbb{Z}_{2}$-graded, while the traces are graded-symmetric and $\mathbb{Z}_{2}$-homogeneous of a common degree which is a characteristic of the model.

It is often the case that $\mathcal{G}$ carries a grading by some larger Abelian group $G$, which we will write in additive notation. The traces of such models are homogeneous of some common degree $-\kappa \in G$, and must satisfy a graded-symmetry condition whose signs involve a group morphism $\epsilon: G \rightarrow \mathbb{Z}_{2}$. In fact, $G$ can be viewed as an extension of $\mathbb{Z}_{2}$ by the kernel of $\epsilon$. To capture this, the formalism of $[1,2]$ can be enhanced as follows. Recall that a $\mathbb{C}$-category $\mathcal{G}$ is called $G$-graded if we are given direct sum decompositions:

$$
\operatorname{Hom}_{\mathcal{G}}(a, b)=\oplus_{g \in G} \operatorname{Hom}_{\mathcal{G}}^{g}(a, b)
$$

for all $a, b \in \mathrm{ObG}$ such the following conditions are satisfied:

1) We have $\operatorname{id}_{a} \in \operatorname{Hom}_{\mathcal{G}}^{0}(a, a)$ for all objects $a$ of $\mathcal{G}$.

2) We have $\operatorname{Hom}_{\mathcal{G}}^{h}(b, c) \circ \operatorname{Hom}_{\mathcal{G}}^{g}(a, b) \subset \operatorname{Hom}_{\mathcal{G}}^{h+g}(a, c)$ for all objects $a, b, c$ and all $g, h \in G$.

\footnotetext{
${ }^{1}$ Recall that $\mathcal{G}$ is called Hom-finite if $\operatorname{dim}_{\mathbb{C}} \operatorname{Hom}_{\mathcal{G}}(a, b)<\infty$ for all $a, b \in \mathrm{Ob \mathcal {G }}$.
} 
Given $u \in \operatorname{Hom}_{\mathcal{G}}^{g}(a, b)$, we set $\operatorname{deg} u:=g$. Then models with a boundary space grading by $G$ can be described as follows:

Definition Let $G$ be an Abelian group, $\epsilon: G \rightarrow \mathbb{Z}_{2}$ a group morphism and $\kappa \in G$ an element of $G$. A graded open 2d TFT of type $(G, \kappa, \epsilon)$ is a pair $(\mathcal{G}, \operatorname{tr})$ where $\mathcal{G}$ is a Hom-finite $G$-graded $\mathbb{C}$-category and $\operatorname{tr}$ is a family of maps $\operatorname{tr}_{a}: \operatorname{Hom}_{\mathcal{G}}(a, a) \rightarrow \mathbb{C}$ defined for all $a \in \mathrm{Ob \mathcal {G }}$, such that:

(1) The following selection rule is satisfied for any homogeneous $u \in \operatorname{Hom}_{\mathcal{G}}(a, a)$ :

$$
\operatorname{tr}_{a}(u)=0 \text { unless } \operatorname{deg} u=\kappa
$$

(2) The following conditions are satisfied for all $a, b \in \mathrm{Ob} \mathcal{G}$ :

$\operatorname{tr}_{a}(v u)=(-1)^{\epsilon(\operatorname{deg} u) \epsilon(\operatorname{deg} v)} \operatorname{tr}_{b}(u v) \forall$ homogeneous $u \in \operatorname{Hom}_{\mathcal{G}}(a, b)$ and $v \in \operatorname{Hom}_{\mathcal{G}}(b, a)$.

(3) For any objects $a, b$ of $\mathcal{G}$ and any $u \in \operatorname{Hom}_{\mathcal{G}}(a, b)$, the following implication holds:

$$
\operatorname{tr}_{a}(v u)=0 \quad \forall v \in \operatorname{Hom}_{\mathcal{G}}(b, a) \Rightarrow u=0
$$

Trivial examples are provided by generic open $2 \mathrm{~d}$ TFTs, with $G=\mathbb{Z}_{2}$ and $\epsilon=\mathrm{id}_{\mathbb{Z}_{2}}$. More complicated models arise by considering an extended worldsheet symmetry group $\Gamma$ which is preserved by the boundary conditions corresponding to the objects of $\mathcal{G}$. Then $\Gamma$ acts on the spaces of boundary and boundary condition-changing observables, i.e. it acts by automorphisms of $\mathcal{G}$ which fix all of its objects. If $\Gamma_{0}$ is the trivially acting subgroup, we have representations of $\Gamma / \Gamma_{0}$ on the vector spaces $\operatorname{Hom}_{\mathcal{G}}(a, b)$. Thus we can view $\mathcal{G}$ as a $\mathbb{C}\left[\Gamma / \Gamma_{0}\right]$-category, where $\mathbb{C}\left[\Gamma / \Gamma_{0}\right]$ is the group algebra of $\Gamma / \Gamma_{0}$. This means that $\operatorname{Hom}_{\mathcal{G}}(a, b)$ are $\mathbb{C}\left[\Gamma / \Gamma_{0}\right]$-modules and the compositions of $\mathcal{G}$ are $\mathbb{C}\left[\Gamma / \Gamma_{0}\right]$ linear. Since the quotient group $\Gamma / \Gamma_{0}$ is Abelian, the spaces $\operatorname{Hom}_{\mathcal{G}}(a, b)$ decompose into one-dimensional irreducible representations, which can be collated together to give decompositions (1.1), where the grading group $G:=\left(\Gamma / \Gamma_{0}\right)^{*}$ is the character group (Pontryaghin dual) of $\Gamma / \Gamma_{0}$. Compatibility of the representations with morphism compositions translates into relations 2) above. On the other hand, the traces satisfy (1.2), where $\kappa \in G$ is dictated by the anomaly of the worldsheet path integral under the action of $\Gamma / \Gamma_{0}$ (the later arises because the action of $\Gamma$ typically involves some nontrivial axial transformation of the worldsheet fermions).

A common application of the above concerns the vector and axial $\mathrm{R}$-symmetries of a sigma model. Recall that two dimensional sigma models with bulk $N=(2,2)$ supersymmetry admit two classes of boundary conditions preserving two supersymmetries, namely boundary conditions of $A$ and $B$-type. Each of these types preserves a certain 
subgroup of the R-symmetry group of the bulk theory. At classical level and in the absence of a bulk superpotential, the bulk theory has vector and axial R-symmetry groups $U(1)_{V}$ and $U(1)_{A}$, each of which can be broken to subgroups already in the bulk. For example, $U(1)_{A}$ is subject to the quantum anomaly and can also be broken classically (to a $\mathbb{Z}_{2}$ subgroup) by a bulk superpotential. On the other hand, $U(1)_{V}$ is preserved by homogeneous bulk superpotentials but is broken by inhomogeneous ones. Let us denote the surviving vector and axial R-symmetry subgroups of the quantum bulk theory by $R_{V}$ and $R_{A}$. Then A-type boundary conditions preserve ${ }^{2} R_{A}$ while breaking $R_{V}$ to a subgroup $R_{V}^{\prime}$. The B-type boundary conditions preserve $R_{V}$, while breaking $R_{A}$ to a subgroup $R_{A}^{\prime}$. Hence the boundary model has a global $R$-symmetry $\Gamma=\Gamma_{A} \times \Gamma_{V}$ where $\Gamma_{A}=R_{A}$ and $\Gamma_{V}=R_{V}^{\prime}$ for A-branes, and $\Gamma_{A}=R_{A}^{\prime}, \Gamma_{V}=R_{V}$ for $B$-branes. As a consequence, the space of boundary observables carries a representation of the Abelian group $\Gamma$. Define $\Gamma^{\prime}=\Gamma / \Gamma_{0}$, where $\Gamma_{0}$ is the trivially acting subgroup. Then the spaces of boundary/boundary condition changing observables carry gradings by the Abelian group of characters $G=\left(\Gamma^{\prime}\right)^{*}$. Upon performing the topological twist, the A (respectively B) -type branes of the untwisted theory become topological D-branes of the A (respectively B) model. Then $\Gamma$ acts on the space of topological observables, which therefore carries a grading by $G$. One thus finds that the corresponding open $2 \mathrm{~d}$ TFT is G-graded.

\section{Graded open 2d TFTs with shifts}

In this section, we fix an Abelian group $G$.

\subsection{Mathematical preparations}

$G$-categories. A $G$-category is a triple $(\mathcal{T}, G, \gamma)$ where $G$ is a group, $\mathcal{T}$ is a $\mathbb{C}$-category and $\gamma$ is a faithful ${ }^{3} G$-action on $\mathcal{T}$, i.e. a group monomorphism $\gamma: G \rightarrow \operatorname{Aut}(\mathcal{T})$, where $\operatorname{Aut}(\mathcal{T})$ is the group of automorphisms of $\mathcal{T}$. Given two $G$-categories, a $G$-invariant functor $(\mathcal{T}, G, \gamma) \rightarrow\left(\mathcal{T}^{\prime}, G, \gamma^{\prime}\right)$ is a functor $F: \mathcal{T} \rightarrow \mathcal{T}^{\prime}$ such that $F \circ \gamma(g)=\gamma^{\prime}(g) \circ F$ for all $g \in G$. Small $G$-categories form a category Cat $_{G}$ whose morphisms are given by $G$-invariant functors.

Graded categories with shifts Given a $G$-graded category $\mathcal{G}$, we let $\operatorname{Aut}^{0}(\mathcal{G})$ denote the group of degree zero automorphisms, i.e. those automorphisms $F$ of $\mathcal{G}$ such that $F\left(\operatorname{Hom}_{\mathcal{G}}^{g}(a, b)\right) \subset \operatorname{Hom}_{\mathcal{G}}^{g}(a, b)$ for all objects $a, b$ and all $g \in G$.

\footnotetext{
${ }^{2}$ For the $A$ model, $R_{A}$ can also suffer a quantum boundary anomaly, so one must chose the A-type boundary conditions such that this anomaly vanishes. This corresponds to the vanishing Maslow index condition in [26].

${ }^{3}$ We restrict to faithful actions in order to avoid pathologies below.
} 
A graded category with shifts is a quadruple $(\mathcal{G}, G, \gamma, s)$ where $\mathcal{G}$ is a $G$-graded category, $\gamma: G \rightarrow \operatorname{Aut}^{0}(\mathcal{G})$ is a group monomorphism and $s(g): \operatorname{id}_{\mathcal{G}} \stackrel{\sim}{\rightarrow} \gamma(g)$ are isomorphisms of functors, subject to the conditions:

$$
s_{a}(g) \in \operatorname{Hom}_{\mathcal{G}}^{-g}(a, \gamma(g)(a))
$$

and:

$$
\gamma(g)\left(s_{a}(h)\right) \circ s_{a}(g)=s_{a}(g+h)
$$

for all $a, b \in \mathrm{Ob} \mathcal{G}$ and $g, h \in G$.

We say that $\gamma(g), s(g)$ are the shifts and suspensions of $\mathcal{G}$. Naturality of $s(g)$ reads:

$$
\gamma(g)(u) \circ s_{a}(g)=s_{b}(g) \circ u
$$

for all $u \in \operatorname{Hom}_{\mathcal{G}}(a, b)$. Combined with (2.2), this gives:

$$
s_{a}(g+h)=s_{\gamma(h)(a)}(g) \circ s_{a}(h)
$$

which in turn implies $s_{a}(e)=\mathrm{id}_{a}$ and $s_{a}(-g)=s_{\gamma(-g)(a)}(g)^{-1}$. Using these equations, one checks the relation:

$$
s_{\gamma(g)(a)}(h)=\gamma(g)\left(s_{a}(h)\right)
$$

which will be useful below.

A morphism $(\mathcal{G}, G, \gamma, s) \rightarrow\left(\mathcal{G}^{\prime}, G, \gamma^{\prime}, s^{\prime}\right)$ of $G$-graded categories with shifts is a functor $F: \mathcal{G} \rightarrow \mathcal{G}^{\prime}$ which preserves morphism degrees, intertwines $\gamma(g)$ and $\gamma^{\prime}(g)$ and maps $s_{a}(g)$ into $s_{F(a)}^{\prime}(g)$ and intertwines $\gamma(g)$ and $\gamma^{\prime}(g)$ (the second condition means that $F:(\mathcal{G}, G, \gamma) \rightarrow\left(\mathcal{G}^{\prime}, G, \gamma^{\prime}\right)$ is a $G$-invariant functor). Small $G$-graded categories with shifts form a category $\operatorname{GrCats}_{G}$ when endowed with the morphisms given by such functors.

The skew category of a $G$-category. The graded skew category of a $G$-category $(\mathcal{T}, G, \gamma)$ is the $G$-graded category $\mathcal{T}^{\bullet}[G]$ having the same objects as $\mathcal{T}$, morphism spaces:

$$
\operatorname{Hom}_{\mathcal{T} \bullet[G]}(a, b) \stackrel{\text { def }}{=} \oplus_{g \in G} \operatorname{Hom}_{\mathcal{T} \bullet[G]}^{g}(a, b)
$$

with :

$$
\operatorname{Hom}_{\mathcal{T} \bullet[G]}^{g}(a, b) \stackrel{\text { def }}{=} \operatorname{Hom}_{\mathcal{T}}(a, \gamma(g)(b))
$$

and morphism compositions given by:

$$
\operatorname{Hom}_{\mathcal{T} \bullet[G]}^{h}(b, c) \times \operatorname{Hom}_{\mathcal{T} \bullet[G]}^{g}(a, b) \ni(v, u) \longrightarrow v * u:=\gamma(g)(v) \circ u \in \operatorname{Hom}_{\mathcal{T} \cdot[G]}^{h+g}(a, b)
$$


The skew category [4] $\mathcal{T}[G]$ of $(\mathcal{T}, G, \gamma)$ is the category obtained from $\mathcal{T}^{\bullet}[G]$ by forgetting the $G$-grading. When the action of $G$ on $\mathrm{Ob} \mathcal{T}$ is free, $\mathcal{T}[G]$ is equivalent [4] with the naive quotient category of $\mathcal{T}$ by $G$.

The fundamental property of $\mathcal{T}[G]$ is that objects belonging to the same $G$-orbit of $\mathcal{T}$ become isomorphic in $\mathcal{T}[G]$. Indeed, $\operatorname{id}_{a} \in \operatorname{Hom}_{\mathcal{T}}(a, a)=\operatorname{Hom}_{\mathcal{T} \bullet[G]}^{-g}(a, \gamma(g)(a))$ and $\operatorname{id}_{\gamma(g)(a)} \in \operatorname{Hom}_{\mathcal{T}}(\gamma(g)(a), \gamma(g)(a)) \approx \operatorname{Hom}_{\mathcal{T} \bullet[G]}^{g}(\gamma(g)(a), a)$ provide mutually inverse isomorphisms between $a$ and $\gamma(g)(a)$. We let $s_{a}(g): a \rightarrow \gamma(g)(a)$ denote the morphism $\operatorname{id}_{a}$ when viewed as an element of $\operatorname{Hom}_{\mathcal{T} \bullet[G]}^{-g}(a, \gamma(g)(a))$.

Notice that $\gamma(g)$ can be viewed as degree zero automorphisms $\gamma^{\bullet}(g)$ of $\mathcal{T}^{\bullet}[G]$, giving a $G$-category structure on the latter. The maps $s_{a}(g)$ give isomorphisms of functors $s(g): \operatorname{id}_{\mathcal{T}[G]} \stackrel{\sim}{\rightarrow} \gamma(g)$, which satisfy $\gamma(g)(s(h)) \circ s(g)=s(g h)$ for all $h, g \in G$. Thus $\left(\mathcal{T}^{\bullet}[G], G, \gamma^{\bullet}, s\right)$ is a graded category with shifts.

Equivariant functors and framings. Given two $G$-categories, $(\mathcal{T}, G, \gamma)$ and $\left(\mathcal{T}^{\prime}, G, \gamma^{\prime}\right)$, a functor $F: \mathcal{T} \rightarrow \mathcal{T}^{\prime}$ is called G-equivariant if there exist isomorphisms of functors $\eta(g): F \circ \gamma(g) \stackrel{\sim}{\rightarrow} \gamma^{\prime}(g) \circ F$ satisfying the compatibility conditions:

$$
\eta\left(g_{1}+g_{2}\right)=\gamma\left(g_{1}\right)\left(\eta\left(g_{2}\right)\right) \circ \eta\left(g_{1}\right)
$$

where we use the obvious slight abuse of notation. These conditions imply that $\eta(0)$ is the identity endomorphism of $F$. Any choice of $\eta$ satisfying (2.9) will be called a framing of $F$. A framed $G$-equivariant endofunctor $F: \mathcal{T} \rightarrow \mathcal{T}^{\prime}$ induces an endofunctor $F^{\bullet}: \mathcal{T}^{\bullet}[G] \rightarrow \mathcal{T}^{\bullet}[G]$ given by $F^{\bullet}(a):=F(a)$ on objects and acting on morphisms as follows:

$u \in \operatorname{Hom}_{\mathcal{T} \bullet[G]}^{g}(a, b)=\operatorname{Hom}_{\mathcal{T}}(a, \gamma(g)(b)) \rightarrow F_{a b}^{\bullet}(u):=\eta_{b}(g) \circ F_{a \gamma(g)(b)}(u) \in \operatorname{Hom}_{\mathcal{T}^{\prime \bullet}[G]}^{g}(F(a), F(b))$

Notice that $F^{\bullet}$ preserves the degree of morphisms.

Obviously, a $G$-invariant functor is $G$-equivariant and admits the trivial framing given by $\eta(g)=$ id for all $g$. Given an invariant functor $F$, we make the convention that $F^{\bullet}$ denotes the functor induced by the trivial framing. Since $G$ is Abelian, each $\gamma(g)$ is $G$-invariant and trivially framed, thus inducing the endofunctor $\gamma^{\bullet}(g)$ of $\mathcal{T}^{\bullet}[G]$ mentioned above.

Given framed $G$-equivariant endofunctors $\left(F, \eta^{F}\right)$ and $\left(G, \eta^{G}\right)$, their composition $F \circ G$ is $G$-equivariant and admits the composite framing $\eta^{F \circ G}(g)=\eta^{F}(g) \circ F\left(\eta^{G}(g)\right)$. We have $(F \circ G)^{\bullet}=F^{\bullet} \circ G^{\bullet}$ when $F \circ G$ is endowed with this framing. Applying this to $G$-invariant functors (which we take to be trivially framed), we find that ${ }^{\bullet}$ gives a functor form $\mathrm{Cat}_{G}$ to $\mathrm{GrCats}_{G}$. 
Orbit categories Given an automorphism $\sigma$ of $\mathcal{T}$, let $G_{\sigma}$ be the subgroup of Aut $(\mathcal{T})$ generated by $\sigma$, which acts tautologically on $\mathcal{T}$ via the inclusion $G_{\sigma} \hookrightarrow \operatorname{Aut}(\mathcal{T})$. We define the orbit category [27] of $\mathcal{T}$ modulo $\sigma$ to be the skew category $\mathcal{T}_{\sigma} \stackrel{\text { def }}{=} \mathcal{T}\left[G_{\sigma}\right]$. We have $G_{\sigma} \approx \mathbb{Z}_{\omega}:=\mathbb{Z} / \omega \mathbb{Z}$, where $\omega=\operatorname{ord} \sigma$ (we set $\omega=0$ when no power of $\sigma$ equals the identity functor). Therefore, $\mathcal{T}_{\sigma}^{\bullet}:=\mathcal{T}^{\bullet}\left[G_{\sigma}\right]$ is $\mathbb{Z}_{\omega}$-graded via $\operatorname{Hom}_{\mathcal{T}_{\sigma}}^{n} \bullet(a, b)=$ $\operatorname{Hom}_{\mathcal{T}}\left(a, \sigma^{n}(b)\right)$ for all $n \in \mathbb{Z}_{\omega}$. An endofunctor $F$ of $\mathcal{T}$ is $G_{\sigma}$-equivariant iff $F \circ \sigma \approx \sigma \circ F$, with strict equality when $F$ is $G_{\sigma}$-invariant.

Notice that $\sigma$ is $G_{\sigma^{-}}$-invariant, thus inducing an automorphism $\sigma^{\bullet}$ of $\mathcal{T}_{\sigma}^{\bullet}$ when endowed with the trivial framing. We have $\sigma^{\bullet} \approx \operatorname{id}_{\mathcal{T}_{\sigma}}$ in $\mathcal{T}_{\sigma}$, via the isomorphism of functors given by $\mathrm{id}_{a} \in \operatorname{Isom}_{\mathcal{T} \bullet[G]}^{-1}(a, \sigma(a))$. Moreover, we have $\operatorname{Hom}_{\mathcal{T}_{\sigma}}^{n}(a, \sigma(b))=$ $\operatorname{Hom}_{\mathcal{T}_{\sigma}}^{n+1}(a, b)$, which shows that $\sigma^{\bullet}$ is a shift functor for the $\mathbb{Z}_{\omega}$-graded category $\mathcal{T}_{\sigma}^{\bullet}$. The application of orbit categories to open $2 \mathrm{~d}$ TFTs was discussed in some detail in Appendix A of [23].

The null restriction of a graded category with shifts Given a graded category with shifts $(\mathcal{G}, G, \gamma, s)$, the non-full subcategory $\mathcal{G}^{0}$ obtained from $\mathcal{G}$ by keeping all objects but restricting to morphisms of trivial degree carries the $G$-action $\gamma^{0}$ obtained by Co-restricting $\gamma$. Hence $\left(\mathcal{G}^{0}, G, \gamma^{0}\right)$ is a $G$-category. Given a morphism $F:(\mathcal{G}, G, \gamma, s) \rightarrow\left(\mathcal{G}^{\prime}, G, \gamma^{\prime}, s^{\prime}\right)$ in $\operatorname{GrCats}_{G}$, we let $F^{0}:\left(\mathcal{G}^{0}, G, \gamma^{0}\right) \rightarrow\left(\mathcal{G}^{\prime 0}, G, \gamma^{\prime 0}\right)$ be the morphism in $\mathrm{Cat}_{G}$ obtained by restricting the action $F$ to morphisms of degree zero. This gives a functor ${ }^{0}: \operatorname{GrCats}_{G} \rightarrow \mathrm{Cat}_{G}$.

Relation between skew categories and graded categories with shifts The following result characterizes skew categories as graded categories with shifts.

Proposition The functors ${ }^{\bullet}: \mathrm{Cat}_{G} \rightarrow \operatorname{GrCats}_{\mathrm{G}}$ and ${ }^{0}: \mathrm{GrCats}_{G} \rightarrow \mathrm{Cat}_{G}$ are mutually quasi-inverse equivalences.

Proof. (1) Given a $G$-category $(\mathcal{T}, G, \gamma)$, we showed above that $\left(\mathcal{T}^{\bullet}[G], G, \gamma^{\bullet}, s\right)$ is a $G$-graded category with shifts. Setting $\mathcal{G}:=\mathcal{T}^{\bullet}[G]$, it is clear that $\mathcal{G}^{0}$ coincides with $\mathcal{T}$ as a $G$-category. Given a morphism $F$ in $\operatorname{Cat}_{G}$, it is also obvious that $\left(F^{\bullet}\right)^{0}=F$. Hence the composition $\left[(-)^{\bullet}\right]^{0}$ is the identity functor of $\mathrm{Cat}_{G}$.

(2) Given a $G$-graded category with $\operatorname{shifts}(\mathcal{G}, G, \gamma, s)$, consider the $G$-category $\left(\mathcal{T}, G, \gamma^{0}\right)$ where $\mathcal{T}:=\mathcal{G}^{0}$. We define a functor of graded categories $\Phi:=\Phi_{\mathcal{G}}: \mathcal{G} \rightarrow$ $\mathcal{T} \bullet[G]$ which acts trivially on objects (i.e. $\Phi(a):=a$ for all $a \in$ ObG) and acts on morphisms as follows:

$$
\operatorname{Hom}_{\mathcal{G}}(a, b) \ni u=\oplus_{g \in g} u_{g} \rightarrow \Phi_{a b}(u):=\oplus_{g \in G} s_{b}(g) \circ u_{g} \in \operatorname{Hom}_{\mathcal{T} \bullet[G]}(a, b)
$$


Here $u_{g} \in \operatorname{Hom}_{\mathcal{G}}^{g}(a, b)$, so $s_{b}(g) \circ u_{g} \in \operatorname{Hom}_{\mathcal{G}}^{0}(a, \gamma(g)(b))=\operatorname{Hom}_{\mathcal{T}}(a, \gamma(g)(b))=\operatorname{Hom}_{\mathcal{T} \bullet[G]}^{g}(a, b)$. To check that $\Phi$ is a functor, pick $u \in \operatorname{Hom}_{\mathcal{G}}^{g}(a, b)$ and $v \in \operatorname{Hom}_{\mathcal{G}}^{h}(b, c)$ and compute:

$$
\begin{aligned}
\Phi_{b c}(v) * \Phi_{a b}(u) & =\gamma(g)\left(s_{c}(h) \circ v\right) \circ s_{b}(g) \circ u=\gamma(g)\left(s_{c}(h)\right) \circ \gamma(g)(v) \circ s_{b}(g) \circ u \\
& =\gamma(g)\left(s_{c}(h)\right) \circ s_{c}(g) \circ v \circ u=s_{c}(g+h) \circ v \circ u=\Phi_{a c}(v \circ u)
\end{aligned}
$$

where we used (2.3) and (2.2). Since $\Phi_{a b}$ are clearly bijective, we find that $\Phi$ is an isomorphism between $\mathcal{G}$ and $\mathcal{T}^{\bullet}[G]=\left(\mathcal{G}^{0}\right)^{\bullet}[G]$.

We now show that $\Phi$ is an isomorphism of graded categories with shifts. To show that $\Phi$ commutes with the $G$-actions, pick $u \in \operatorname{Hom}_{\mathcal{G}}^{h}(a, b)$ and compute:

$$
\Phi\left(\gamma^{\bullet}(g)(u)\right)=s_{\gamma(g)(b)}(h) \circ \gamma^{\bullet}(g)(u)=\gamma(g)\left(s_{b}(h)\right) \circ \gamma^{\bullet}(g)(u)=\gamma(g)(\Phi(u))
$$

where we used (2.5). Finally, we have $\Phi\left(s_{a}(g)\right)=s_{\gamma(g)(a)}(-g) \circ s_{a}(g)=s_{a}(e)=\mathrm{id}_{a}$, where $\operatorname{id}_{a}$ is viewed as an element of $\operatorname{Hom}_{\mathcal{T} \bullet[G]}^{-g}(a, \gamma(g)(a))$ as required by the definition of $\Phi$. Thus $\Phi\left(s_{a}(g)\right)=s_{a}^{\mathcal{T}^{\bullet}[G]}(g)$ is the corresponding suspension of $\mathcal{T}^{\bullet}[G]$. We conclude that $\Phi$ is an isomorphism of graded categories with shifts. Given a morphism $F$ : $(\mathcal{G}, G, \gamma, s) \rightarrow\left(\mathcal{G}^{\prime}, G, \gamma^{\prime}, s^{\prime}\right)$ in $\operatorname{GrCats}_{G}$, it is easy to check the identity $\Phi_{\mathcal{G}^{\prime}} \circ F=$ $\left(F^{0}\right)^{\bullet} \circ \Phi_{\mathcal{G}}$, which shows that $\Phi_{\mathcal{G}}$ give an isomorphism of functors $\operatorname{id}_{\operatorname{GrCats}_{G}} \rightarrow\left[(-)^{0}\right]^{\bullet}$.

The conclusion now follows by combining (1) and (2).

The proposition allows us to reconstruct a graded category with shifts $\mathcal{G}$ as the skew category of its null restriction $\mathcal{G}^{0}$, thereby reducing the theory of graded categories with shifts to that of $G$-categories. Notice that the functor $\Phi_{\mathcal{G}}$ realizing this correspondence is determined by the suspensions of $\mathcal{G}$. In the next subsection, we show how this result can be used to express the data of certain graded open $2 d$ TFTs in terms of $G$-categories with supplementary structure.

Convention In order to simplify notation, we will often use the same symbol for the $G$-representation $\gamma$ of a $G$-category $\mathcal{T}$ and the representation $\gamma^{\bullet}$ of its skew category $\mathcal{T}^{\bullet}[G]$. Which one is meant should be clear from the context.

\subsection{G-graded open 2d TFTs with shifts}

Let $G$ be an Abelian group as before. G-graded open 2d topological field theories can be described through ungraded categories provided that they admit a $G$-action with special properties. Such special theories can be described as follows.

Definition A graded open 2d TFT with shifts is a system $(\mathcal{G}, G, \gamma, s, \operatorname{tr}, \kappa, \epsilon)$ such that: 
(1) $(\mathcal{G}, G, \gamma, s)$ is a Hom-finite $G$-graded $\mathbb{C}$-category with shifts.

(2) $(\mathcal{G}, \operatorname{tr})$ is a graded $2 \mathrm{~d}$ open TFT of type $(G, \kappa, \epsilon)$, where $\kappa \in G$ and $\epsilon \in$ $\operatorname{Hom}\left(G, \mathbb{Z}_{2}\right)$

(3) The traces $\operatorname{tr}_{a}: \operatorname{Hom}_{\mathcal{G}}(a, a) \rightarrow \mathbb{C}$ satisfy the equivariance conditions:

$$
\operatorname{tr}_{\gamma(g)(a)}(\gamma(g)(u))=(-1)^{\epsilon(g)(\epsilon(\kappa)+1)} \operatorname{tr}_{a}(u)
$$

for all $a \in \mathrm{ObG}, u \in \operatorname{Hom}_{\mathcal{G}}(a, a)$ and $g \in G$.

We will see in a moment that this structure is equivalent with the one given in the definition below.

Definition Let $(\mathcal{T}, G, \gamma)$ be a Hom-finite $G$-category and fix an element $\kappa \in G$ and a group morphism $\epsilon: G \rightarrow \mathbb{Z}_{2}$. A G-equivariant cyclic structure of type $(\kappa, \epsilon)$ on $(\mathcal{T}, G, \gamma)$ is a family of linear maps $\operatorname{tr}_{a}: \operatorname{Hom}_{\mathcal{T}}(a, \gamma(\kappa)(a)) \rightarrow \mathbb{C}$ defined for all $a \in \mathrm{Ob} \mathcal{T}$ which obey the symmetry conditions:

$$
\operatorname{tr}_{a}(v \circ u)=\operatorname{tr}_{b}(\gamma(\kappa)(u) \circ v) \quad \forall u \in \operatorname{Hom}_{\mathcal{T}}(a, b) \quad \forall v \in \operatorname{Hom}_{\mathcal{T}}(b, \gamma(\kappa)(a))
$$

the equivariance conditions:

$$
\operatorname{tr}_{\gamma(g)(a)}(\gamma(g)(u))=(-1)^{\epsilon(g)(\epsilon(\kappa)+1)} \operatorname{tr}_{a}(u) \quad \forall u \in \operatorname{Hom}_{\mathcal{T}}(a, \gamma(\kappa)(a))
$$

and the following nondegeneracy condition for all $u \in \operatorname{Hom}_{\mathcal{T}}(a, b)$ :

$$
\operatorname{tr}_{a}(v \circ u)=0 \quad \forall v \in \operatorname{Hom}_{\mathcal{T}}(b, \gamma(k)(a)) \Rightarrow u=0 .
$$

We also say that $(\mathcal{T}, G, \gamma, t r, \kappa, \epsilon)$ is an equivariant cyclic category of type $(G, \gamma, \kappa, \epsilon)$.

Observation Let $(\mathcal{T}, G, \gamma, t r, \kappa, \epsilon)$ be an equivariant cyclic category and set $S:=$ $\gamma(\kappa)$. Using the traces $\operatorname{tr}_{a}$, define linear maps $\phi_{a b}: \operatorname{Hom}_{\mathcal{T}}(a, b) \rightarrow \operatorname{Hom}_{\mathcal{T}}(b, S(a))^{\mathrm{v}}$ via: $\phi_{a b}(u)(v):=\operatorname{tr}_{a}(v \circ u)$. Then the pair $(S, \phi)$ is a Serre functor on $\mathcal{T}$ in the sense of [3]. Indeed, the symmetry condition (2.11) amounts to naturality of $\phi_{a b}$ in $a$ and $b$, while the nondegeneracy condition (2.13) amounts to bijectivity of $\phi_{a b}$. The traces can be recovered as $\operatorname{tr}_{a}=\phi_{a a}\left(\mathrm{id}_{a}\right)$. Since $G$ is Abelian, the functor $S=\gamma(\kappa)$ is $G$-invariant. On the other hand, condition (2.12) becomes:

$$
\phi_{\gamma(g)(a), \gamma(g)(a)}\left(\operatorname{id}_{\gamma(a)}\right) \circ \gamma_{a S(a)}(g)=(-1)^{\epsilon(g)(\epsilon(\kappa)+1)} \phi_{a a}\left(\operatorname{id}_{a}\right)
$$

which can be viewed as an equivariance condition for $\phi$. Hence $(S, \phi)$ is a rather special type of equivariant Serre functor on $\mathcal{T}$. A $G$-equivariant cyclic category can be viewed 
as a $G$-category endowed with such an equivariant Serre functor. A general discussion of equivariant Serre functors can be found in Appendix A.

The following results shows that graded open $2 \mathrm{~d}$ TFTs with shifts are equivalent with equivariant cyclic categories. This translates the physics formalism into a subset of the theory of equivariant Serre functors.

Proposition The following data are equivalent:

(a) A graded open $2 \mathrm{~d}$ TFT with shifts $(\mathcal{G}, G, \gamma, s, \operatorname{tr}, \kappa, \epsilon)$

(b) A $G$-equivariant cyclic category $(\mathcal{T}, G, \gamma, t r, \kappa, \epsilon)$.

Moreover, the relation between (a) and (b) is given by $\mathcal{G}=\mathcal{T}^{\bullet}[G]$ (thus $\mathcal{T}=\mathcal{G}^{0}$ ) and $\operatorname{tr}_{a}(u)=\operatorname{tr}_{a}(u)$ for all $u \in \operatorname{Hom}_{\mathcal{T} \bullet[G]}^{g}(a, a)=\operatorname{Hom}_{\mathcal{T}}^{g-\kappa}(a, \gamma(\kappa)(a))$.

Proof.

(1) Given the data at (a), set $\mathcal{T}=\mathcal{G}^{0}$ and define maps $\operatorname{tr}_{a}: \operatorname{Hom}_{\mathcal{T}}(a, \gamma(\kappa)(a)) \rightarrow \mathbb{C}$ via:

$$
\operatorname{tr}_{a}(u):=\operatorname{tr}_{a}\left(\Phi^{-1}(u)\right) \quad \forall u \in \operatorname{Hom}_{\mathcal{T}}(a, \gamma(\kappa)(a))=\operatorname{Hom}_{\mathcal{T} \bullet[G]}^{\kappa}(a, a)
$$

where $\Phi: \mathcal{G} \stackrel{\sim}{\rightarrow} \mathcal{T}^{\bullet}[G]$ is the isomorphism of graded categories with shifts constructed in the proof of the last proposition of Subsection 2.1.

Given $u \in \operatorname{Hom}_{\mathcal{T}}(a, b)=\operatorname{Hom}_{\mathcal{T} \bullet[G]}^{0}(a, b)$ and $v \in \operatorname{Hom}_{\mathcal{T}}(b, \gamma(\kappa)(a))=\operatorname{Hom}_{\mathcal{T}}^{\kappa} \cdot[G](b, a)$, we have:

$$
\begin{array}{r}
\operatorname{tr}_{a}(v \circ u)=\operatorname{tr}_{a}\left(\Phi^{-1}(v * u)\right)=\operatorname{tr}_{a}\left(\Phi^{-1}(v) \Phi^{-1}(u)\right)=\operatorname{tr}_{b}\left(\Phi^{-1}(u) \Phi^{-1}(v)\right) \\
=\operatorname{tr}_{b}\left(\Phi^{-1}(u * v)\right)=\operatorname{tr}_{b}\left(\Phi^{-1}(\gamma(\kappa)(u) \circ v)\right)=\operatorname{tr}_{b}(\gamma(\kappa)(u) \circ v)
\end{array}
$$

where we used $\epsilon(\operatorname{deg} u)=\epsilon(0)=0$. This shows that (2.11) is satisfied. Equation (2.12) follows trivially from (2.10). It is also clear that $t r_{a}$ are nondegenerate.

(2) Given the data at (b), set $\mathcal{G}:=\mathcal{T}^{\bullet}[G]$ and define maps $\operatorname{tr}_{a}: \operatorname{Hom}_{\mathcal{G}}^{\kappa}(a, a) \rightarrow \mathbb{C}$ via:

$$
\operatorname{tr}_{a}(u):=\operatorname{tr}_{a}(u) \quad \forall u \in \operatorname{Hom}_{\mathcal{G}}^{\kappa}(a, a)=\operatorname{Hom}_{\mathcal{T}}(a, \gamma(\kappa)(a))
$$

with extension by zero to other degrees. It is clear that $\operatorname{tr}_{a}$ are nondegenerate and satisfy the selection rule (1.2) as well as (2.10). To show (1.3), pick $u \in \operatorname{Hom}_{\mathcal{G}}^{g}(a, b)=$ $\operatorname{Hom}_{\mathcal{T}}(a, \gamma(g)(b))$ and $v \in \operatorname{Hom}_{\mathcal{G}}^{h}(b, a)=\operatorname{Hom}_{\mathcal{T}}(b, \gamma(h)(a))$ with $g+h=\kappa$. Then

$\operatorname{tr}_{a}(v * u)=\operatorname{tr}_{a}(\gamma(g)(v) \circ u)=\operatorname{tr}_{\gamma(g)(b)}(\gamma(\kappa)(u) \circ \gamma(g)(v))=\operatorname{tr}_{\gamma(g)(b)}(\gamma(g+h)(u) \circ$ $\gamma(g)(v))=\operatorname{tr}_{\gamma(g)(b)}(\gamma(g)(\gamma(h)(u) \circ v))=(-1)^{\epsilon(g)(\epsilon(\kappa)+1)} \operatorname{tr}_{b}(\gamma(h)(u) \circ v)=(-1)^{\epsilon(g) \epsilon(h)} \operatorname{tr}_{b}(u *$ $v)$, 
where we used (2.11) and (2.12) and noticed that $(-1)^{\epsilon(g)(\epsilon(\kappa)+1)}=(-1)^{\epsilon(g) \epsilon(h)}$ since $g+h=\kappa$ implies $\epsilon(\kappa)=\epsilon(g)+\epsilon(h)$.

Trivial examples Consider a cyclic group $G=\mathbb{Z}_{\omega}:=\mathbb{Z} / \omega \mathbb{Z}$ (notice that we allow for the case $s=0$, in which case $G=\mathbb{Z})$ and let $[1]:=\gamma(1)$. Setting $[n]:=\gamma(n)=[1]^{n}$, we have $s(n)=s(1)^{n}$ where we use an obvious abuse of notation. The positive integer $\omega$ is the order of the automorphism [1] (we set ord[1] $=0$ if $[1]^{n} \neq \mathrm{id}_{\mathcal{G}}$ for all $n$ ). Thus a $\mathbb{Z}_{\omega}$-graded category with shifts is simply a $\mathbb{Z}_{\omega}$-graded category endowed with an automorphism [1] of order $\omega$ and a degree -1 isomorphism of functors $s(1): \operatorname{id}_{\mathcal{G}} \stackrel{\sim}{\rightarrow}[1]$. The later induces linear isomorphisms $\operatorname{Hom}_{\mathcal{G}}^{k}(a, b[1]) \approx \operatorname{Hom}_{\mathcal{G}}^{k+1}(a, b)$ for all objects $a, b$. The element $\kappa \in \mathbb{Z}_{\omega}$ is the common degree of the traces $\operatorname{tr}_{a}$. On the other hand, a $\mathbb{Z}_{\omega}$-cyclic category is simply a category $\mathcal{T}$ endowed with an automorphism [1] of order $\omega$ and such that $([\kappa], \phi)$ is a Serre functor, where the maps $\phi_{a b}: \operatorname{Hom}_{\mathcal{T}}(a, b) \rightarrow \operatorname{Hom}_{\mathcal{T}}(b, a[\kappa])^{\mathrm{v}}$ correspond to cyclic nondegenerate traces subject

to the equivariance condition $t r_{a[1]} u[1]=(-1)^{\epsilon(1)(\epsilon(\kappa)+1)} \operatorname{tr}_{a}(u)$. Thus $\mathcal{T}$ is a Calabi-Yau category of dimension $\kappa$, whose shift functor has order $\omega$ and whose traces are equivariant in the sense above (this refines results of [29]). The proposition above shows that such categories are the same thing as $\mathbb{Z}_{\omega}$-graded open $2 \mathrm{~d}$ TFTs with shifts, the relation in this case being given by taking the orbit category of $\mathcal{T}$ with respect to the shift functor [1]. Notice that the correct grading group of the TFT can be recovered as the subgroup of $\operatorname{Aut}(\mathcal{T})$ generated by [1]. Two familiar cases are $s=2$ and $s=0$, with morphism $\epsilon$ given by $\mathrm{id}_{\mathbb{Z}_{2}}$ and $\bmod 2$ reduction respectively. The first case corresponds to a generic open $2 \mathrm{~d}$ TFT, while the second describes $\mathbb{Z}$-graded theories, such as those associated with the bounded derived category of coherent sheaves on a generic compact Calabi-Yau manifold. This last case was discussed in Appendix A of [23].

\section{G-graded dG categories}

In open topological field theory, $\mathbb{Z}$-graded associative categories often appear as total cohomology categories of differential graded $(\mathrm{dG})$ categories. In this section, we extend this construction to the $G$-graded case. After a brief general discussion, we construct a $\mathrm{dG}$ category with shifts whose objects are graded integrable modules 'with extended grading' over a curved differential graded algebra. A particular case of this construction will allow us to give a description of graded D-branes in Landau-Ginzburg models which manifestly displays the grading by characters of the surviving group of vector and axial R-symmetries. 
Let $G$ be an Abelian group endowed with a pairing (=group morphism) $\cdot: G \times G \rightarrow$ $\mathbb{Z}_{2}$ and fix an element $\delta \in G$.

Definition A graded dG category of type $(G, \delta)$ is a $G$-graded $\mathbb{C}$-category $\mathcal{D}$ whose morphism spaces are endowed with linear maps $d_{a b}: \operatorname{Hom}_{\mathcal{D}}(a, b) \rightarrow \operatorname{Hom}_{\mathcal{D}}(a, b)$ which are homogeneous of degree $\delta$ and satisfy the following conditions:

(1) $d_{a b}^{2}=0$ for all $a, b \in \mathrm{ObD}$

(2) $d_{a c}(v u)=d_{b c}(v) u+(-1)^{\delta \cdot \operatorname{deg} v} v d_{a b}(u)$ for all homogeneous $u \in \operatorname{Hom}_{\mathcal{D}}(a, b)$ and $v \in \operatorname{Hom}_{\mathcal{D}}(b, c)$.

Notice that the definition makes use of the pairing $\cdot: G \times G \rightarrow \mathbb{Z}_{2}$. In the application to open $2 \mathrm{~d}$ TFTs, this morphism will have the form:

$$
g_{1} \cdot g_{2}:=\epsilon\left(g_{1}\right) \epsilon\left(g_{2}\right)
$$

where $\epsilon: G \rightarrow \mathbb{Z}_{2}$ is the group morphism used in Section 1 and in the right hand side we use the ring multiplication of $\mathbb{Z}_{2}$.

We define the total cohomology category $H(\mathcal{D})$ to be the $G$-graded category having the same objects as $\mathcal{D}$ and morphism spaces given by $\operatorname{Hom}_{H(\mathcal{D})}(a, b):=\oplus_{g \in G} \operatorname{Hom}_{H(\mathcal{D})}^{g}(a, b)$, where:

$$
\operatorname{Hom}_{H(\mathcal{D})}^{g}(a, b):=\left[\operatorname{ker}\left(d_{a b}\right) \cap \operatorname{Hom}_{\mathcal{D}}^{g}(a, b)\right] /\left[d_{a b}\left(\operatorname{Hom}_{\mathcal{D}}^{g-\delta}(a, b)\right)\right] .
$$

Given any closed morphism $\phi \in \operatorname{Hom}_{\mathcal{D}}(a, b)$, we let $\phi^{H} \in \operatorname{Hom}_{H(\mathcal{D})}(a, b)$ be the induced morphism in $H(\mathcal{D})$. We also let $Z(\mathcal{D})$ be the category obtained from $\mathcal{D}$ by keeping only closed morphisms.

A twisted $d G$ automorphism of $\mathcal{D}$ is a pair $(F, \chi)$ such that $\chi$ is a non-vanishing complex number and $F$ is an automorphism of the underlying associative category, which satisfies the condition:

$$
F_{a b}\left(d_{a b}(u)\right)=\chi d_{F(a) F(b)}\left(F_{a b}(u)\right)
$$

for all morphisms $u \in \operatorname{Hom}_{\mathcal{D}}(a, b)$. Twisted $\mathrm{dG}$ automorphisms form a group with the composition rule $(F, \chi) \circ\left(F^{\prime}, \chi^{\prime}\right)=\left(F \circ F^{\prime}, \chi \chi^{\prime}\right)$ (the identity element is $\left.\left(\operatorname{id}_{\mathcal{D}}, 1\right)\right)$. We will denote this group by $\operatorname{Aut}_{\text {tw }}(\mathcal{D})$. Notice that any twisted dG automorphism $F$ of $\mathcal{D}$ induces an automorphism $F^{H}$ of $H(\mathcal{D})$ by passage to cohomology.

Definition A $d G$ category with shifts is a system $(\mathcal{D}, G, \delta, \gamma, s)$ such that:

(1) $\mathcal{D}$ is a graded dG category of type $(G, \delta)$

(2) $\gamma: G \rightarrow \operatorname{Aut}_{\mathrm{tw}}(\mathcal{D})$ is a group monomorphism.

(3) $s(g): \operatorname{id}_{\mathcal{D}} \stackrel{\sim}{\rightarrow} \gamma(g)$ are closed isomorphisms of functors subject to conditions $(2.1)$ and $(2.2)$ (with $\mathcal{G}$ replaced with $\mathcal{D}$ ). 
The closure condition on $s(g)$ means $d_{a, \gamma(g)(a)}\left(s_{a}(g)\right)=0$ for all $a \in \mathrm{Ob} \mathcal{D}$ and all $g \in G$. This implies ${ }^{4}$ that $s_{a}(g)$ descend to isomorphisms $s_{a}^{H}(g)$ in $H(\mathcal{D})$.

It is easy to see that the total cohomology category $H(\mathcal{D})$ of a graded dG category with shifts is a graded category with shifts, whose shift functors and suspensions $\gamma^{H}, s^{H}$ are induced from $\gamma, s$ by passage to cohomology. More precisely, $\left(H(\mathcal{D}), G, \gamma^{H}, s^{H}\right)$ is a G-graded category with shifts.

\subsection{Integrable modules over a curved differential graded algebra}

In this subsection, we discuss a class of graded $d G$ categories which generalizes a construction considered in [20]. As we will see below, this affords a 'manifestly symmetric' description of the $\mathrm{dG}$ category of graded D-branes in Landau-Ginzburg models.

Modules with extended grading over a graded algebra Let $G_{V}$ be an Abelian group and $B$ a $G_{V}$-graded associative algebra. Given another Abelian group $G$ and a morphism $\psi: G_{V} \rightarrow G$, a right extended-graded module of type $(G, \psi)$ over $B$ is a right $B$-module $M$ endowed with a vector space decomposition:

$$
M=\oplus_{g \in G} M^{g},
$$

such that $M^{g} B^{k} \subset M^{g+\psi(k)}$ for all $g \in G$ and $k \in G_{V}$. Given such a module, we set $\operatorname{deg}(m)=g$ for $m \in M^{g}$. A decomposition (3.3) with this property will be called a extended graded structure of type $(G, \psi)$ on $M$. Given two such modules $M, N$, we define:

$$
\operatorname{Hom}_{B}^{g}(M, N):=\left\{f \in \operatorname{Hom}_{B}(M, N) \mid f\left(M^{h}\right) \subset M^{g+h} \quad \forall h \in G\right\}
$$

and $\operatorname{Hom}_{B}^{\mathrm{gr}}(M, N):=\oplus_{g \in G} \operatorname{Hom}_{B}^{g}(M, N)$. This gives a $G$-graded category denoted $\mathrm{Gr}_{B}^{G, \psi}$. Notice that $\mathrm{Gr}_{B}^{G_{V}, \mathrm{id}_{G_{V}}}$ coincides with the usual category of graded $B$-modules.

Twist functors and suspensions. Given $g \in G$, we let $(g) \in \operatorname{Aut}^{0}\left[\operatorname{Gr}_{B}^{G, \psi}\right]$ denote the twist functor by $(g)$. This acts on objects via $M(g)^{h}:=M^{h+g}$ and takes a morphism $u \in \operatorname{Hom}_{B}^{h}(M, N)$ into the same map $u$ but viewed as an element of $\operatorname{Hom}_{B}^{h}(M(g), N(g))$. We have $(g) \circ(h)=(g+h)$ for all $g, h \in G$, as well as the relation $\operatorname{Hom}_{B}^{h}(M, N(g))=$ $\operatorname{Hom}_{B}^{h+g}(M, N)$.

Let $s_{M}(g)$ be the $g$-th suspension morphism of $M$, i.e. the identity morphism of $a$ viewed as an element of $\operatorname{Hom}_{B}^{-g}(M, M(g))$. These maps define isomorphisms of

\footnotetext{
${ }^{4}$ It is easy to check that the inverse of any closed and homogeneous isomorphism $\phi$ is closed and homogeneous of opposite degree, and induces the inverse of $\phi^{H}$ on cohomology.
} 
functors $s(g): \mathrm{id}_{\mathrm{Gr}_{B}^{G, \psi}} \stackrel{\sim}{\rightarrow}(g)$. Setting $\tilde{\gamma}(g):=(g)$, it is clear that $(2.2)$ holds. Thus $\left(\operatorname{Gr}_{B}^{G, \psi}, G, \tilde{\gamma}, s\right)$ is a graded category with shifts. We let $\operatorname{gr}_{B}^{G, \psi}$ denote the full subcategory of $\mathrm{Gr}_{B}^{G, \psi}$ consisting of finitely-generated modules. This is again a graded category with shifts.

Free extended-graded modules An extended-graded module $M \in \mathrm{Ob}\left[\mathrm{Gr}_{B}^{(G, \psi)}\right]$ will be called free if it admits a basis $e_{s}$ all of whose elements are $G$-homogeneous. In this case, we have a decomposition:

$$
M=\oplus_{s} e_{s} B
$$

and

$$
M^{g}=\oplus_{s, k: g_{s}+\psi(k)=g} e_{s} B^{k}
$$

Change of grading. The collection of pairs $(G, \psi)$ where $G$ is a group and $\psi$ : $G_{V} \rightarrow G$ is a group morphism forms a category $\Lambda$ whose morphisms $(G, \psi) \rightarrow\left(G^{\prime}, \psi^{\prime}\right)$ are maps $\mu: G \rightarrow G^{\prime}$ such that $\mu \circ \psi=\psi^{\prime}$. Given $\mu \in \operatorname{Hom}_{\Lambda}\left((G, \psi),\left(G^{\prime}, \psi^{\prime}\right)\right)$ and $M$ in $\operatorname{Gr}_{B}^{G, \psi}$, we let $\mu_{*}(M)$ be the object of $\operatorname{Gr}_{B}^{G^{\prime}, \psi^{\prime}}$ defined by the decomposition $\mu_{*}(M)=\oplus_{g^{\prime} \in G^{\prime}} \mu_{*}(M)^{g^{\prime}}$, where $\mu_{*}(M)^{g^{\prime}}:=\oplus_{g \in G: \mu(g)=g^{\prime}} M^{g}$ (it is easy to check that the condition $\mu^{*}(M)^{g^{\prime}} B^{k} \subset \mu^{*}(M)^{g^{\prime}+\psi^{\prime}(k)}$ is satisfied). Notice that we set $\mu_{*}(M)^{g^{\prime}}=0$ if $g^{\prime} \notin \mu(G)$. It is clear that a homogeneous morphism $u \in \operatorname{Hom}_{\operatorname{Gr}_{B}^{G, \psi}}^{h}(M, N)$ satisfies $u\left(\mu_{*}(M)^{g^{\prime}}\right) \subset \mu_{*}(M)^{g^{\prime}+\mu(h)}$, and we let $\mu_{*}(u) \in \operatorname{Hom}_{\operatorname{Gr}_{B}^{G^{\prime}, \psi^{\prime}}}^{\mu(h)}\left(\mu_{*}(M), \mu_{*}(N)\right)$ be the morphism of $\operatorname{Gr}_{B}^{G^{\prime}, \psi^{\prime}}$ obtained in this manner. Then $\mu_{*}$ gives a functor from $\mathrm{Gr}_{B}^{G, \psi}$ to $\operatorname{Gr}_{B}^{G^{\prime}, \psi^{\prime}}$. Given composable morphisms $\mu, \nu$ in $\Lambda$, one has $(\mu \circ \nu)_{*}=\mu_{*} \circ \nu_{*}$. Further, the identity automorphism of $(G, \psi)$ induces the identity automorphism of $\operatorname{Gr}_{B}^{G, \psi}$. These observations imply that any isomorphism $\mu:(G, \psi) \rightarrow\left(G^{\prime}, \psi^{\prime}\right)$ of $\Lambda$ induces an isomorphism of categories $\mu_{*}: \mathrm{Gr}_{B}^{G, \psi} \rightarrow \mathrm{Gr}_{B}^{G^{\prime}, \psi^{\prime}}$, so we can identify the categories of graded modules defined by isomorphic pairs $(G, \psi)$.

Extensions of grading. Consider a $G_{V}$-graded algebra $B$, and let $B^{\#}$ be the associative algebra obtained from $B$ by forgetting the grading. Fixing an Abelian group

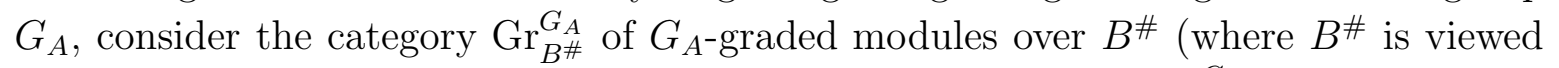
as a graded algebra concentrated in degree zero). The objects of $\operatorname{Gr}_{B_{A}^{\#}}^{G_{A}}$ are $B^{\#}$-modules $M$ endowed with a decomposition of the form $M=\oplus_{\alpha \in G_{A}} M_{\alpha}$ into $B^{\#}{ }_{-}$submodules.

Consider a short exact sequence:

$$
\text { (E) } 0 \rightarrow G_{V} \stackrel{\psi}{\rightarrow} G \stackrel{\epsilon}{\rightarrow} G_{A} \rightarrow 0
$$

representing an extension class $\xi \in \operatorname{Ext}_{\mathbb{Z}}^{1}\left(G_{A}, G_{V}\right)$. Notice that $\operatorname{Gr}_{B}^{G_{A}}=\operatorname{Gr}_{B}^{\left(G_{A}, 0\right)}$, where 0 is the null morphism from $G_{V}$ to $G_{A}$. Since $\epsilon \circ \mu=0$, we can view $\epsilon$ as a 
morphism in $\operatorname{Hom}_{\Lambda}\left((G, \psi),\left(G_{A}, 0\right)\right)$. This induces a functor $\epsilon_{*}: \operatorname{Gr}_{B}^{(G, \psi)} \rightarrow \operatorname{Gr}_{B}^{G_{A}}$. Since $\epsilon$ is surjective, we have $\epsilon_{*}(M)=M$ as vector spaces, and we set $M_{\alpha}:=\epsilon_{*}(M)^{\alpha}=$ $\oplus_{g \in G: \epsilon(g)=\alpha} M^{g}$ for all $\alpha \in G_{A}$.

Given an object $M$ of $\mathrm{Gr}_{B_{A}^{\#}}^{G_{A}}$, an extension of grading of $M$ along the exact sequence $(E)$ is an extended graded module structure $M=\oplus_{g \in G} M^{g}$ of type $(G, \psi)$ such that $M_{\alpha}=\oplus_{\epsilon(g)=\alpha} M^{g}$ for all $\alpha \in G_{A}$, i.e. such that the $\epsilon_{*}$-image of the extended-graded $B$-module obtained in this manner recovers $M$. Such a structure exists iff $M$ belongs to the image of the functor $\epsilon_{*}: \mathrm{Gr}_{B}^{(G, \psi)} \rightarrow \mathrm{Gr}_{B^{\#}}^{G_{A}}$, in which case we say that $M$ is gradable along (E).

Recall that extensions of $G_{A}$ by $G_{V}$ form a category. Given another extension $\left(E^{\prime}\right) \quad 0 \rightarrow G_{V} \stackrel{\psi^{\prime}}{\rightarrow} G^{\prime} \stackrel{\epsilon^{\prime}}{\rightarrow} G_{A} \rightarrow 0$, the morphisms $(E) \rightarrow\left(E^{\prime}\right)$ are given by maps $\mu: G \rightarrow G^{\prime}$ such that $\mu \circ \psi=\psi^{\prime}$ and $\epsilon^{\prime} \circ \mu=\epsilon$. In particular, $\mu$ is a morphism in $\Lambda$ from $(G, \psi)$ to $\left(G^{\prime}, \psi^{\prime}\right)$, so it induces a functor $\mu_{*}: \operatorname{Gr}_{B}^{G, \psi} \rightarrow \operatorname{Gr}_{B}^{G^{\prime}, \psi^{\prime}}$. We thus have a triple of functors $\left(\mu_{*}, \epsilon_{*}, \epsilon_{*}^{\prime}\right)$ which satisfy $\epsilon_{*}^{\prime} \circ \mu_{*}=\epsilon_{*}$, and $\mu_{*}$ is an isomorphism of categories when $\mu$ is an isomorphism of groups. When $\mu$ is an isomorphism, this shows that $\epsilon_{*}$ and $\epsilon_{*}^{\prime}$ can be identified consistently with the identification of $\operatorname{Gr}_{B}^{G, \psi}$ and $\operatorname{Gr}_{B}^{G^{\prime}, \psi^{\prime}}$ via $\mu_{*}$. It follows that extensions of grading are essentially determined by the extension class $\xi$ of the sequence $(E)$.

Component description. Given $M \in \mathrm{Ob}\left[\mathrm{Gr}_{B_{A}^{\#}}^{G_{A}}\right]$ endowed with an extension of grading along $(E)$, the $G_{A}$-components $M_{\alpha}$ become graded $B$-modules via the following construction. Let us pick elements $g_{\alpha} \in G$ such that $\epsilon\left(g_{\alpha}\right)=\alpha$ for each $\alpha \in G_{A}$. Then the $\epsilon$-preimage of $\alpha$ consists of the elements $g=g_{\alpha}+\psi(k)$ with $k \in G_{V}$. Defining:

$$
M_{\alpha}^{k}:=M^{g_{\alpha}+\psi(k)} \forall \alpha \in G_{A}, \forall k \in G_{V}
$$

it is clear that $M_{\alpha}^{k} B^{l} \subset M_{\alpha}^{k+l}$. Thus the decomposition $M_{\alpha}=\bigoplus_{g \in G: \epsilon(g)=\alpha} M^{g}=$ $\oplus_{k \in G_{V}} M_{\alpha}^{k}$ gives a $G_{V}$-grading of the module $M_{\alpha}$, and $M$ can be viewed as direct sum of graded $B$-modules. Given another choice of elements $g_{\alpha}^{\prime} \in G$ such that $\epsilon\left(g_{\alpha}^{\prime}\right)=\alpha$, we have $g_{\alpha}^{\prime}-g_{\alpha}=\psi\left(k_{\alpha}\right)$ for some $k_{\alpha} \in G_{V}$, so the $B$-graded module structures $\left(M^{\prime}\right)_{\alpha}^{k}=M^{g_{\alpha}^{\prime}+\psi(k)}$ determined by this new choice on $M_{\alpha}$ are related to (3.7) via the twists:

$$
M_{\alpha}^{\prime}=M_{\alpha}\left(k_{\alpha}\right)
$$

It follows that the $G_{V}$-gradings on $M_{\alpha}$ are determined by the $G$-grading on $M$ up to independent twists. We stress that the gradings on $M_{\alpha}$ depend on the choice of $g_{\alpha}$.

Conversely, any collection $\left(M_{\alpha}\right)_{\alpha \in G_{A}}$ of $G_{V}$-graded modules defines an object $M:=$ $\oplus_{\alpha \in G_{A}} M_{\alpha}$ of $\mathrm{Gr}_{B_{A}^{\#}}^{G_{A}}$ which is (E)-gradable for any fixed extension (3.6). To see this, pick elements $g_{\alpha} \in G$ such that $\epsilon\left(g_{\alpha}\right)=\alpha$. Given $g \in G$, set $\alpha:=\epsilon(g)$. Then 
$g-g_{\alpha} \in \operatorname{ker} \epsilon=\operatorname{im} \psi$, so there exists a unique $k \in G_{V}$ such that $g=g_{\alpha}+\psi(k)$. This shows that the map $(k, \alpha) \in G_{V} \times G_{A} \stackrel{f}{\rightarrow} \psi(k)+g_{\alpha} \in G$ is a bijection. Defining $M^{g}:=M_{\alpha}^{k}$ where $(k, \alpha)=f^{-1}(g)$, we can thus write the linear subspace decomposition $M=\oplus_{\alpha \in G_{A}, k \in G_{V}} M_{\alpha}^{k}$ as $M=\oplus_{g \in G} M^{g}$. It is clear that the condition $M_{\alpha}^{k} B^{l} \subset M_{\alpha}^{k+l}$ implies $M^{g} B^{l} \subset M^{g+\psi(l)}$. Thus $M$ endowed with this $G$-grading is an extended graded module of type $(G, \psi)$ over $B$, and $\epsilon_{*}(M)$ recovers the original object of $\operatorname{Gr}_{B *}^{G_{A}}$. If we pick other elements $g_{\alpha}^{\prime} \in \epsilon^{-1}(\{\alpha\})$, then $g_{\alpha}^{\prime}-g_{\alpha}=\psi\left(k_{\alpha}\right)$ for some $k_{\alpha} \in G_{V}$ and we find that the $G$-grading determined by $g_{\alpha}^{\prime}$ is related to that given by $g_{\alpha}$ via:

$$
M^{\prime g}=M^{g-\psi\left(k_{\epsilon(g)}\right)} .
$$

Hence the choice of the extension $(E)$ determines the $G$-grading on $M$ up such transformations. This discussion establishes the following:

Proposition The following statements are equivalent for any object $M$ of $\mathrm{Gr}_{B \#}^{G_{A}}$ :

(a) $M$ is gradable along some extension $(E)$ of type (3.6)

(b) $M$ is gradable along any extension (E) of type (3.6)

(c) Each of the $B^{\#}$-submodules $M_{\alpha}$ admits a structure of graded $B$-module.

In this case, the extension $(E)$ together with the graded $B$-module structures on $M_{\alpha}$ determine the $G$-grading on $M$ up to transformations of the form (3.9), while ( $E$ ) and the $G$-grading on $M$ determines the $G_{V^{-}}$-gradings on $M_{\alpha}$ up to independent twists of the form (3.8).

The proposition shows that the notion of module with extended grading is essentially the same as a collection of graded $B$-modules. The gain afforded by the former language is that it manifestly displays the larger group $G$.

Component description of free extended-graded modules Consider an extendedgraded module of type $(G, \psi)$ over $B$ and fix an extension $(E)$ as in (3.6). Also pick elements $g_{\alpha} \in G$ such that $\epsilon\left(g_{\alpha}\right)=\alpha$ for all $\alpha \in G_{A}$. Then it is easy to see that $M$ is free as an extended-module iff all of the graded $B$-modules $M_{\alpha}$ determined by $g_{\alpha}$ are free. We leave the details as an exercise for the reader.

Curved differential graded algebras. Consider an Abelian group $G_{V}$ endowed with a pairing $\cdot: G_{V} \times G_{V} \rightarrow \mathbb{Z}_{2}$. A $G_{V}$-graded curved differential graded algebra $(\mathrm{CdGA})$ over $\mathbb{C}$ is a triple $(B, d, c)$ where $B$ is a $G_{V}$-graded associative algebra over $\mathbb{C}$, $c$ is a homogeneous element of $B$ and $d$ is a homogeneous derivation of $B$ such that $d^{2}(b)=[c, b]$ for all $b \in B$, where $\left[b_{1}, b_{2}\right]:=b_{1} b_{2}-(-1)^{\operatorname{deg} b_{1} \cdot \operatorname{deg} b_{2}} b_{2} b_{1}$ denotes the graded commutator (computed using the pairing on $G_{V}$ ). If $c \neq 0$, then we let $h \in G_{V}$ denote 
the degree of $c$. The Leibniz condition for the derivation $d$ takes the form:

$$
d\left(b_{1} b_{1}\right)=d\left(b_{1}\right) b_{2}+(-1)^{\operatorname{deg}(d) \cdot \operatorname{deg}\left(b_{1}\right)} b_{1} d\left(b_{2}\right)
$$

Notice that we must have $2 \operatorname{deg}(d)=h$ unless $c=0$ or $d$ is identically zero. When $c=0$, the pair $(B, d)$ is a $G_{V}$-graded differential algebra (a $G_{V}$-graded differential category with one object). The case $d=0$ is also special. In this case, $(B, 0, c)$ is a curved dGA iff $c$ is a homogeneous central element of $B$, since the condition $d^{2}(b)=[c, b]$ becomes $[c, b]=0$ for all $b \in B$. Then (3.10) is trivially satisfied and there are no constraints on the degree of $c$.

Integrable modules over a curved dGA. Let us fix an Abelian group $G$ with a pairing : : $G \times G \rightarrow \mathbb{Z}_{2}$ and a group morphism $\psi: G_{V} \rightarrow G$. Also fix an element $\delta \in G$. A connection of degree $\delta$ on $M \in \mathrm{Ob}\left[\operatorname{gr}_{B}^{G, \psi}\right]$ is a $\mathbb{C}$-linear map $D: M \rightarrow M$ satisfying the homogeneity condition:

$$
\operatorname{deg} D(m)=\operatorname{deg} m+\delta
$$

and the Leibniz identity:

$$
D(m b)=D(m) b+(-1)^{\delta \cdot \operatorname{deg} m} m d(b) .
$$

These relations require $\delta=\psi(\operatorname{deg} d)$ unless $d=0$, in which case $\delta$ is unconstrained.

An integrable module of type $(G, \psi, \delta)$ over $B$ is a pair $\mathbb{M}:=(M, D)$ such that $M$ is a finitely-generated right extended-graded $B$-module of type $(G, \psi)$ and $D$ is a connection of degree $\delta$ on $M$, subject to the condition:

$$
D^{2}(m)=m c \quad \forall m \in M
$$

This equation requires $2 \delta=\psi(h)$, which is compatible with the previous constraint $\delta=\psi(\operatorname{deg} d)$ when $d \neq 0$ (since $2 \operatorname{deg} d=h$ in that case). Notice that (3.13) constrains $\delta$ also for the case $d=0$.

It is easy to check that integrable modules of type $(G, \psi, \delta)$ form a dG category $\mathrm{DG}_{c}^{G, \psi, \delta}(B)$ of type $(G, \delta)$, with morphism spaces given by $\operatorname{Hom}_{\mathrm{DG}_{c}^{G, \psi, \delta}(B)}(\mathbb{M}, \mathbb{N}):=$ $\operatorname{Hom}_{B}^{\mathrm{gr}}(M, N)$ and differentials defined through $d_{\mathbb{M N}}(u):=D_{N} \circ u-(-1)^{\delta \cdot \operatorname{deg} u} u \circ D_{M}$ for homogeneous $u \in \operatorname{Hom}_{B}^{\mathrm{gr}}(M, N)$.

A particular case A particularly simple case arises when the differential on $B$ vanishes (thus $c$ must be a homogeneous central element of $B$, and we assume $c \neq 0$ ). In this case, $D$ is a homogeneous module endomorphism of $M$ of degree $\delta$ which satisfies (3.13); this requires $2 \delta=\psi(h)$ where $h=\operatorname{deg} c$. 
The twist functors of $\operatorname{gr}_{B}^{G, \psi}$ induce twist functors on $\mathrm{DG}_{c}^{G, \psi, \delta}(B)$ as follows. For any object $\mathbb{M}=(M, D)$, we set $\mathbb{M}(g):=(M(g), D(g))$, where $D(g)$ is the result of acting on the module endomorphism $D$ with the twist functor of $\operatorname{gr}_{B}^{G, \psi}$. We let $(g)$ act on the morphisms of $\mathrm{DG}_{c}^{G, \psi, \delta}(B)$ in the same way as in $\mathrm{Gr}_{B}^{G, \psi}$. It is easy to check that $(g)$ are dG-automorphisms, making $\mathrm{DG}_{c}^{G, \psi, \delta}(B)$ into a dG category of type $(G, \delta)$.

The maps $s_{\mathbb{M}}(g):=s_{M}(g) \in \operatorname{Hom}_{B}^{-g}(M, M(g))=\operatorname{Hom}_{\mathrm{DG}_{c}^{G, \psi, \delta}(B)}^{-g}(\mathbb{M}, \mathbb{M}(g))$ give isomorphisms of functors from $\mathrm{id}_{\mathrm{DG}_{c}^{G, \psi, \delta}(B)}$ to $(g)$. In general, these isomorphisms are not closed, so they cannot be used to make $\mathrm{DG}_{c}^{G, \psi, \delta}(B)$ into a graded category with shifts. Indeed, they satisfy $D_{M(g)} \circ s_{M}(g)=s_{M}(g) \circ D_{M}$ rather than the closure condition $d_{\mathbb{M M}(g)}\left(s_{M}(g)\right)=D_{M(g)} \circ s_{M}(g)-(-1)^{\delta \cdot g} s_{M}(g) \circ D_{M}=0$. This can be remedied in the following situation, which will be relevant below.

Let us assume given an exact sequence of the form (3.6) with $G_{A}=\mathbb{Z}_{2}$ and consider the pairing on $G$ given in (3.1). Define automorphisms $\gamma(g)$ of the underlying associative category of $\mathrm{DG}_{c}^{G, \psi, \delta}(B)$ via:

$$
\gamma(g)(M, D)=\left(M(g),(-1)^{\epsilon(g) \epsilon(\delta)} D(g)\right)
$$

and:

$$
\gamma_{\mathbb{M N}}(g)(u):=u(g) \quad \forall u \in \operatorname{Hom}_{\mathrm{DG}_{c}^{G, \psi, \delta}(B)}(\mathbb{M}, \mathbb{N}) .
$$

We have $\gamma\left(g_{1}\right) \gamma\left(g_{2}\right)=\gamma\left(g_{1}+g_{2}\right)$ for all $g_{1}, g_{2} \in G$. Then $\gamma(g)$ are twisted dG functors, namely they satisfy:

$$
d_{\gamma(g)(\mathbb{M}) \gamma(g)(\mathbb{N})} \circ \gamma_{\mathbb{M N}}(g)=\chi(g) \gamma_{\mathbb{M N}}(g) \circ d_{\mathbb{M N}}
$$

for the character $\chi(g)=(-1)^{\epsilon(g) \epsilon(\delta)}$ of $G$. On the other hand, the maps $s_{\mathbb{M}}(g)=s_{M}(g)$ are closed when viewed as elements of $\operatorname{Hom}_{\mathrm{DG}_{c}^{G, \psi, \delta}(B)}^{-g}(\mathbb{M}, \gamma(g)(\mathbb{M}))$, and we find that $\left(\mathrm{DG}_{c}^{G, \psi, \delta}(B), G, \delta, \gamma, s\right)$ is a dG category with shifts. Passing to the total cohomology category $\mathcal{G}:=H\left(\mathrm{DG}_{c}^{G, \psi, \delta}(B)\right)$, we have induced automorphisms $\gamma^{\mathcal{G}}(g)$ of $\mathcal{G}$ and morphisms of functors $s^{\mathcal{G}}(g): \operatorname{id}_{\mathcal{G}} \rightarrow \gamma^{\mathcal{G}}(g)$ of degree $-g$ making $\left(\mathcal{G}, G, \gamma^{\mathcal{G}}, s^{\mathcal{G}}\right)$ into a graded category with shifts.

Consider the $\mathrm{dG}$ category $\mathrm{DG}_{c}^{\mathbb{Z}_{2}, 0, \epsilon(\delta)}(B)$, where 0 is the null morphism form $G_{V}$ to $G_{A}=\mathbb{Z}_{2}$. This consists of pairs $\mathbb{M}=(M, D)$ with $\left.M \in \mathrm{Ob}_{\left[g_{B \#}^{\mathbb{Z}}\right.}^{\mathbb{Z}_{2}}\right]$ and $D \in \operatorname{End}_{B^{\#}}(M)$ a homogeneous module endomorphism of degree $\epsilon(\delta)$ satisfying $D^{2}=c$ (recall that $B^{\#}$ is concentrated in degree zero). The homogeneity condition on $D$ means $D\left(M_{\alpha}\right)=$ $M_{\alpha+\epsilon(\delta)}$. The functor $\epsilon_{*}: \mathrm{Gr}_{B}^{G, \psi} \rightarrow \mathrm{Gr}_{B^{\#}}^{\mathbb{Z}_{2}}$ induces a dG functor $\tilde{\epsilon}_{*}: \mathrm{DG}_{c}^{G, \psi, \delta}(B) \rightarrow$ $\mathrm{DG}_{c}^{\mathbb{Z}_{2}, 0, \epsilon(\delta)}(B)$, defined through $\tilde{\epsilon}_{*}(M, D)=\left(\epsilon_{*}(M), \epsilon_{*}(D)\right)$ on objects and $\tilde{\epsilon}_{*}(u):=\epsilon_{*}(u)$ on morphisms. An object of $\operatorname{DG}_{c}^{\mathbb{Z}_{2}, 0, \epsilon(\delta)}(B)$ is called gradable along $(E)$ if it lies in the image of this functor. Picking elements $g_{\alpha} \in G$ such that $\epsilon\left(g_{\alpha}\right)=\alpha$, consider the 
$G_{V}$-grading of $M_{\alpha}$ defined as in the previous subsection. Then it is easy to see that gradability of $\mathbb{M}$ reduces to gradability of $M$ (i.e. the condition that $M$ are graded $B$-modules) and the condition that the components $D_{\alpha, \alpha+\epsilon(\delta)} \in \operatorname{Hom}_{B}\left(M_{\alpha}, M_{\alpha+\epsilon(\delta)}\right)$ of $D$ are $G_{V^{-}}$homogeneous of degree $\delta+g_{\alpha}-g_{\alpha+\epsilon(\delta)}$.

\section{Application to graded B-type branes in Landau-Ginzburg models}

In this section, we reconsider the case of graded B-type branes in Landau-Ginzburg models, giving a complete treatment of the vector and axial gradings. While this was already studied in $[9,10,12]$ from various perspectives, we will see in a moment that the grading induced by vector and axial R-symmetries on the corresponding open $2 \mathrm{~d}$ TFT is a bit subtle. The reason is that the total grading arises from a group extension of the axial $\mathbb{Z}_{2}$-grading by the vector $\mathbb{Z}$-grading, and this extension is nontrivial when the degree of the bulk superpotential is odd. We will also give a complete description of the open 2d TFT defined by graded D-branes, as well as its precise relation with the triangulated category of matrix factorizations considered in [10]. Using this correspondence will allow us to extract a precise proposal for the Serre functor on the latter.

Consider the B-twisted topological Landau-Ginzburg model with target $\mathbb{C}^{n}$ and coordinate ring $B:=\mathbb{C}\left[\phi_{1} \ldots \phi_{n}\right]$. The $U(1)$ vector R-symmetry induces a $G_{V}=$ $U(1)^{*}=\mathbb{Z}$-grading of $B$ with $\operatorname{deg} \phi_{i}=q_{i}$, where we take $q_{i}$ to be integral (see Appendix $\mathrm{B}$ for the worldsheet realization). From the worldsheet perspective, this corresponds to a normalization in which the bulk superpotential $W \in B$ has integral degree $h$. We endow the group $G_{V}=\mathbb{Z}$ with the trivial pairing $m \cdot n=0$ (this is insures that the graded commutator in $B$ is the usual commutator). Then the bulk model is encoded by the commutative $\mathbb{Z}$-graded curved dGA $(B, 0, W)$, which has trivial differential.

\subsection{The dG category of graded D-branes}

As explained in Appendix B, the axial R-symmetry is broken by the bulk superpotential to a subgroup $\Gamma_{A} \approx \mathbb{Z}_{2}$, so the surviving vector-axial R-symmetry group is $\Gamma=\Gamma_{V} \times$ $\Gamma_{A}=U(1) \times \mathbb{Z}_{2}$. It turns out that the subgroup $\Gamma_{0}$ which acts trivially on the boundary data depends on the parity of $h$. Namely, $\Gamma_{0}$ is trivial for even $h$, while for odd $h$ one finds that $\Gamma_{0}$ is a $\mathbb{Z}_{2}$ subgroup. As a consequence, the faithfully acting group $\Gamma_{h}^{\prime}:=\Gamma / \Gamma_{0}$ is given by:

$$
\Gamma_{h}^{\prime}= \begin{cases}U(1) \times \mathbb{Z}_{2} & \text { for } h \in 2 \mathbb{N} \\ U(1) & \text { for } h \in 2 \mathbb{N}+1\end{cases}
$$


with group of characters:

$$
G_{h}=\left(\Gamma_{h}^{\prime}\right)^{*}= \begin{cases}\mathbb{Z} \times \mathbb{Z}_{2} & \text { for } h \in 2 \mathbb{N} \\ \mathbb{Z} & \text { for } h \in 2 \mathbb{N}+1\end{cases}
$$

When $h$ is odd, the vector and axial gradings on the spaces of boundary and boundary condition changing observables combine into a single $\mathbb{Z}$-grading and thus they cannot be treated as independent. One has a group extension:

$$
0 \rightarrow \mathbb{Z} \stackrel{\psi_{h}}{\rightarrow} G_{h} \stackrel{\epsilon_{h}}{\rightarrow} \mathbb{Z}_{2} \rightarrow 0
$$

which is trivial only for even $h$. Here $G_{V}=\mathbb{Z}$ and $G_{A}=\mathbb{Z}_{2}$. The morphisms $\psi_{h}, \epsilon_{h}$ are given by (see Appendix B):

$$
\psi_{h}(k)=\left\{\begin{array}{ll}
(k, 0) & \text { for } h \in 2 \mathbb{N} \\
2 k & \text { for } h \in 2 \mathbb{N}+1
\end{array} .\right.
$$

and

$$
\left\{\begin{array}{ll}
\epsilon_{h}(k, \alpha)=\alpha & \text { for } h \in 2 \mathbb{N} \\
\epsilon_{h}(k)=k(\bmod 2) & \text { for } h \in 2 \mathbb{N}+1
\end{array} .\right.
$$

We will use the pairing (3.1) on the group $G_{h}$.

The most general topological D-branes of the model form the the full dG category $\mathrm{DG}_{W}\left(B^{\#}\right)$ of $\mathrm{DG}_{W}^{\mathbb{Z}_{2}, 0, \hat{1}}(B)$ consisting of free graded $B^{\#}$-modules (recall that $B^{\#}$ is viewed as a graded algebra concentrated in degree zero). This is the well-known dG category of matrix factorizations discussed in [24, 25].

As shown in Appendix B, 'graded topological D-branes' (i.e. those topological D-branes which preserve the vector R-symmetry) correspond to objects of $\mathrm{DG}_{W}\left(B^{\#}\right)$ which are freely extended-graded along the extension (4.2). More precisely, such branes are described by integrable $B$-modules $\mathbb{M}=(M, D)$ of type $\left(G_{h}, \psi_{h}, \delta_{h}\right)$, where $M$ is free as an extended-graded module and:

$$
\delta_{h}:=\operatorname{deg}(D)=\left\{\begin{array}{ll}
\left(\frac{h}{2}, \hat{1}\right) \in \mathbb{Z} \times \mathbb{Z}_{2} & \text { for } h \in 2 \mathbb{N}, \\
h \in \mathbb{Z} & \text { for } h \in 2 \mathbb{N}+1
\end{array} .\right.
$$

Notice that $\epsilon_{h}\left(\delta_{h}\right)=\hat{1}$, and $2 \delta_{h}=\psi_{h}(h)$, as required. Since $(B, 0, W)$ has trivial differential, $D$ is a simply a module endomorphism of $M$ of $G_{h}$-degree $\delta_{h}$, which satisfies $D^{2}=W$. This gives the following description, which is quite similar in spirit to that used in $[13,14,16,20]$ for the case of B-type branes on Calabi-Yau manifolds. We can take it as a mathematical definition: 
The $d G$ category of graded D-branes defined by the $L G$ model with bulk data $(B, W)$ is the full $d G$ subcategory $\mathrm{DG}_{W}^{\mathrm{gr}}(B)$ of $\mathrm{DG}_{W}^{G_{h}, \psi_{h}, \delta_{h}}(B)$ consisting of free extended-graded modules.

Notice that this description manifestly displays the vector-axial grading group $G_{h}$. The total cohomology category $\mathcal{G}:=H\left(\mathrm{DG}_{W}^{\mathrm{gr}}(B)\right)$ is the category of boundary sectors. The dG category $\mathrm{DG}_{W}^{\mathrm{gr}}(B)$ is a dG category with shifts of type $\left(G_{h}, \delta_{h}, \gamma_{h}, s_{h}\right)$, where the twisted $\mathrm{dG}$ automorphisms $\gamma_{h}(g)$ are defined as in (3.14) and the suspensions $s_{h}(g)$ are as described in the previous section. These descend to automorphisms $\gamma_{h}^{\mathcal{G}}(g)$ of $\mathcal{G}$ and morphisms of functors $s_{h}^{\mathcal{G}}(g): \operatorname{id}_{\mathcal{G}} \rightarrow \gamma^{\mathcal{G}}(g)$, making $\mathcal{G}$ into a graded category with shifts of type $\left(G_{h}, \gamma_{h}^{\mathcal{G}}, s_{h}^{\mathcal{G}}\right)$. Its null restriction $\mathcal{T}:=\mathcal{G}^{0}=H^{0}\left(\mathrm{DG}_{W}^{\mathrm{gr}}(B)\right)$ is the triangulated category of matrix factorizations considered in [10] and [12].

\subsection{Shift functor and distinguished twist functor}

Consider the functors $T_{h}:=\gamma_{h}\left(g_{T}\right), \tau_{h}:=\gamma_{h}\left(g_{\tau}\right)$ and $\rho_{h}=\gamma_{h}\left(g_{\rho}\right)$, where $g_{T}, g_{\tau}$ and $g_{\rho}$ are given by:

$$
\left\{\begin{array}{llll}
g_{\tau}=(1, \hat{0}), & g_{T}=\left(\frac{h}{2}, \hat{1}\right), & g_{\rho}=(0, \hat{1}) & \text { for } h \in 2 \mathbb{N}, \\
g_{\tau}=2, & g_{T}=h, & g_{\rho}=1 & \text { for } h \in 2 \mathbb{N}+1
\end{array}\right.
$$

We have $g_{T}=\delta_{h}, \epsilon_{h}\left(g_{\tau}\right)=\hat{0}$ and $\epsilon_{h}\left(g_{T}\right)=\epsilon_{h}\left(g_{\rho}\right)=\hat{1}$. Notice the relation $g_{T}^{2}=g_{\tau}^{h}$, which implies

$$
T_{h}^{2}=\left(\tau_{h}\right)^{h}
$$

as well as the relation $g_{T}=g_{\rho}+\left[\frac{h}{2}\right] g_{\tau}$, which gives

$$
T_{h}=\rho_{h} \circ\left(\tau_{h}\right)^{\left[\frac{h}{2}\right]} .
$$

Here [ ] denotes the integer part. Using these equations, it is easy to check that the subgroup of $\operatorname{Aut}_{\mathrm{tw}}\left(\mathrm{DG}_{W}^{\mathrm{gr}}(B)\right)$ generated by $T_{h}$ and $\tau_{h}$ is isomorphic with $G_{h}$. In fact, the representation $\gamma_{h}$ takes the form:

$$
\begin{cases}\gamma_{h}(k, \alpha)=\tau_{h}^{k} \rho_{h}^{\alpha}=T_{h}^{\alpha} \tau_{h}^{k-\frac{\alpha h}{2}} & \text { for } h \in 2 \mathbb{N} \\ \gamma_{h}(k)=\rho_{h}^{k}=T_{h}^{k} \tau_{h}^{-\frac{k(h-1)}{2}} & \text { for } h \in 2 \mathbb{N}+1\end{cases}
$$

Passing to zeroth cohomology, the functor $T_{h}$ induces the shift functor $T_{h}^{\mathcal{T}}=[1]$ of the triangulated category $\mathcal{T}$. It also turns out that functor $\tau_{h}^{\mathcal{T}}$ induced by $\tau_{h}$ on $\mathcal{T}$ coincides with the 'distinguished' twist functor considered in [10, 12] (see the last subsection below). 


\subsection{Traces}

Given an object $\mathbb{M}=(M, D)$ of $\mathrm{DG}_{W}^{\text {gr }}(B)$, the free graded module $M$ can be decomposed as:

$$
M=\oplus_{s} e_{s} B
$$

where $e_{s}$ is a $G$-homogeneous basis of $M$ with $\operatorname{deg} e_{s}=g_{s} \in G$. With respect to such a basis, a module endomorphism $u \in \operatorname{Hom}_{B}(M, M)$ has a matrix with entries $u_{s t} \in B$ determined by the decomposition:

$$
u\left(e_{s}\right)=\sum_{t} e_{t} u_{t s}
$$

This allows one to define a linear map $\operatorname{Str}_{\mathbb{M}}: \operatorname{Hom}_{\mathrm{DG}_{W}^{\mathrm{gr}}(B)}(\mathbb{M}, \mathbb{M}) \rightarrow B$ via:

$$
\operatorname{Str}_{\mathbb{M}}(u):=\sum_{s}(-1)^{\epsilon_{h}\left(g_{s}\right)} u_{s s}
$$

When $u$ is homogeneous of degree $g \in G$, we have $u_{t s}=0$ unless $\psi_{h}\left(\operatorname{deg} u_{t s}\right)=g+g_{s}-g_{t}$. This implies $\psi_{h}\left(\operatorname{deg}\left(\operatorname{Str}_{\mathbb{M}}(u)\right)=g\right.$ unless $\operatorname{Str}_{\mathbb{M}}(u)=0$. In particular, we have $\operatorname{Str}_{\mathbb{M}}(u)=$ 0 unless $\epsilon(g)=\hat{0}$. It is easy to check the relations:

$$
\operatorname{Str}_{\mathbb{M}}(u v)=(-1)^{\epsilon_{h}(\operatorname{deg} u) \epsilon_{h}(\operatorname{deg} v)} \operatorname{Str}_{\mathbb{N}}(v u)
$$

for homogeneous $u \in \operatorname{Hom}_{\mathrm{DG}_{W}^{\mathrm{gr}}(B)}(\mathbb{N}, \mathbb{M})$ and $v \in \operatorname{Hom}_{\mathrm{DG}_{W}^{\mathrm{gr}}(B)}(\mathbb{M}, \mathbb{N})$, as well as the relation:

$$
\operatorname{Str}_{\mathbb{M}(g)}(u(g))=(-1)^{\epsilon_{h}(g)} \operatorname{Str}_{\mathbb{M}}(u)
$$

where $(g)$ is the twist by $g$.

The category $Z\left(\mathrm{DG}_{W}^{\mathrm{gr}}(B)\right)$ is endowed with the traces of $[5,8]$ :

$$
\operatorname{tr}_{\mathbb{M}}(u):=\oint \frac{d \phi_{1} \ldots d \phi_{n}}{(2 \pi i)^{n}} \frac{\operatorname{Str}_{\mathbb{M}}\left[(\partial D)^{n} u\right]}{\partial_{1} W \ldots \partial_{n} W} \quad\left(u \in Z\left(\operatorname{Hom}_{\mathrm{DG}_{W}^{\mathrm{gr}}}(\mathbb{M}, \mathbb{M})\right)\right)
$$

where $\mathbb{M}=(M, D)$ and $(\partial D)^{n}:=\partial_{1} D \ldots \partial_{n} D$. Notice that $\partial_{i} D \in \operatorname{Hom}_{B}^{\delta_{h}-\psi_{h}\left(q_{i}\right)}(M, M)$, so $\operatorname{deg}(\partial D)^{n}=n \delta_{h}-\psi_{h}\left(\sum_{i=1}^{n} q_{i}\right)$. The traces are easily seen to satisfy $\operatorname{tr}_{\mathbb{M}}\left(d_{\mathbb{M} \mathbb{M}}(u)\right)=0$ as well as

$$
\operatorname{tr}_{\mathbb{M}}(u v)=(-1)^{\epsilon_{h}(\operatorname{deg} u) \epsilon_{h}(\operatorname{deg} v)} \operatorname{tr}_{\mathbb{N}}(v u)
$$

for homogeneous $u \in \operatorname{Hom}_{\mathrm{DG}_{W}^{\mathrm{gr}}(B)}(\mathbb{N}, \mathbb{M})$ and $v \in \operatorname{Hom}_{\mathrm{DG}_{W}^{\mathrm{gr}}(B)}(\mathbb{M}, \mathbb{N})$. They also satisfy the selection rule:

$$
\operatorname{tr}_{\mathbb{M}}(u)=0 \text { unless } \operatorname{deg}(u)=\kappa_{h}
$$


where $\kappa_{h} \in G_{h}$ is given by $\kappa_{h}=n \delta_{h}-\psi_{h}\left(\sum_{i=1}^{n} q_{i}\right)$, i.e.:

$$
\kappa_{h}= \begin{cases}\left(\frac{h}{2} \hat{c}, n \bmod 2\right)=\left(\frac{n h}{2}-\sum_{i=1}^{n} q_{i}, \quad n \bmod 2\right) \in \mathbb{Z} \times \mathbb{Z}_{2} & \text { for } h \in 2 \mathbb{N}, \\ h \hat{c}=n h-2 \sum_{i=1}^{n} q_{i} \in \mathbb{Z} & \text { for } h \in 2 \mathbb{N}+1\end{cases}
$$

Here:

$$
\hat{c}=n-\frac{2}{h} \sum_{i=1}^{n} q_{i}
$$

is the conformal anomaly. To establish (4.14), notice that the integral $\oint \frac{d \phi_{1} \ldots d \phi_{n}}{(2 \pi i)^{n}} f\left(\phi_{1} \ldots \phi_{n}\right)$ of a homogeneous rational function of $\phi_{1} \ldots \phi_{n}$ vanishes unless $f$ has degree $-\sum_{i=1}^{n} q_{i}$. Using this observation, it follows that (4.12) vanishes for homogeneous $u$ unless $\operatorname{deg}\left(\operatorname{Str}_{\mathbb{M}}\left[(\partial D)^{n} u\right]\right)=$ $\operatorname{deg}\left(\partial_{1} W \ldots \partial_{n} W\right)-\sum_{i=1}^{n} q_{i}=n h-2 \sum_{i=1}^{n} q_{i}$. Since $\psi_{h}\left(\operatorname{deg} \operatorname{Str}_{\mathbb{M}}\left[(\partial D)^{n} u\right]\right)=\psi_{h}\left(\operatorname{deg}\left[(\partial D)^{n} u\right]\right)$ when the left hand side is nonzero, we find that (4.12) vanishes unless $\psi_{h}(\operatorname{deg} u)=$ $\psi_{h}\left(n h-2 \sum_{i=1}^{n} q_{i}\right)-\psi_{h}\left(\operatorname{deg}\left[(\partial D)^{n}\right]\right)=n\left(\psi_{h}(h)-\delta_{h}\right)-\psi_{h}\left(\sum_{i=1}^{n} q_{i}\right)=\kappa_{h}$, where we used the relation $\psi_{h}(h)=2 \delta_{h}$.

Finally, notice that equation (4.11) implies the relation:

$$
\operatorname{tr}_{\mathbb{M}(g)} u(g)=(-1)^{\epsilon_{h}(g)} \operatorname{tr}_{\mathbb{M}}(u),
$$

which is equivalent with the equivariance conditions (recall the definition (3.14)) :

$$
\operatorname{tr}_{\gamma_{h}(g)(\mathbb{M})} \gamma_{h}(g)(u)=(-1)^{\epsilon_{h}(g)\left(\epsilon_{h}\left(\kappa_{h}\right)+1\right)} \operatorname{tr}_{\mathbb{M}}(u)
$$

where we noticed that $\epsilon_{h}\left(n \delta_{h}\right)=\epsilon_{h}\left(\kappa_{h}\right)=n(\bmod 2)$. Setting $g=g_{\tau}$ or $g=g_{T}$, the last equation gives:

$$
\operatorname{tr}_{\tau_{h}(\mathbb{M})}\left(\tau_{h}(u)\right)=\operatorname{tr}_{\mathbb{M}}(u), \quad \operatorname{tr}_{T_{h}(\mathbb{M})} T_{h}(u)=(-1)^{n+1} \operatorname{tr}_{\mathbb{M}}\left(T_{h}(u)\right) .
$$

\subsection{The graded topological field theory with shifts}

The traces (4.12) descend to the total cohomology category $\mathcal{G}$, inducing maps which we denote by $\operatorname{tr}^{\mathcal{G}}$. Assuming that $\operatorname{tr}^{\mathcal{G}}$ are nondegenerate, the discussion above shows that $\left(\mathcal{G}, G_{h}, \gamma_{h}^{\mathcal{G}}, s_{h}^{\mathcal{G}}, \operatorname{tr}^{\mathcal{G}}, \kappa_{h}, \epsilon_{h}\right)$ is a graded open $2 \mathrm{~d}$ TFT with shifts in the sense of Section 2.

Consider the triangulated category $\mathcal{T}=\mathcal{G}^{0}$ of graded matrix factorizations. We let $\gamma_{h}^{\mathcal{T}}$ be the functor induced from $\gamma_{h}^{\mathcal{G}}$ by restriction to morphisms of degree zero. As in Section 2.2, $\mathcal{T}$ carries traces $\operatorname{tr}_{\mathbb{M}}^{\mathcal{T}}: \operatorname{Hom}_{\mathcal{T}}\left(\mathbb{M}, \gamma_{h}(\kappa)(\mathbb{M})\right) \rightarrow \mathbb{C}$ induced from $\mathcal{G}$, which make it into a graded cyclic category. Equations (4.15) and (4.9) imply that the Serre functor $S_{h}=\gamma_{h}\left(\kappa_{h}\right)$ defined by this cyclic structure takes the form:

$$
S_{h}=\left\{\begin{array}{ll}
\tau_{h}^{\frac{h \hat{c}}{2}} \rho_{h}^{n}=T_{h}^{n} \tau_{h}^{\frac{h}{2}(\hat{c}-n)} & \text { for } h \in 2 \mathbb{N} \\
\rho_{h}^{h \hat{c}}=T_{h}^{h \hat{c}} \tau_{h}^{-\frac{h(h-1)}{2} \hat{c}} & \text { for } h \in 2 \mathbb{N}+1
\end{array} .\right.
$$


As in Section 2, the graded category with shifts $\mathcal{G}$ can be recovered as the skew category $\mathcal{G}=\mathcal{T}^{\bullet}\left[G_{h}\right]$. Combining everything, we conclude:

Assume that (4.12) is homologically nondegenerate, i.e. the trace $\operatorname{tr}^{\mathcal{G}}$ induced on $\mathcal{G}$ is nondegenerate. Then the system $\left(\mathcal{G}, G_{h}, \gamma_{h}^{\mathcal{G}}, s_{h}^{\mathcal{G}}, \operatorname{tr}^{\mathcal{G}}, \kappa_{h}, \epsilon_{h}\right)$ is a graded open $2 d$ TFT with shifts and the corresponding system $\left(\mathcal{T}, G_{h}, \gamma_{h}^{\mathcal{T}}, \operatorname{tr}^{\mathcal{T}}, \kappa_{h}, \epsilon_{h}\right)$ is an equivariant cyclic category. In particular, (4.20) is an equivariant Serre functor on $\mathcal{T}$ under this assumption, when considered together with the isomorphisms $\operatorname{Hom}_{\mathcal{T}}(\mathbb{M}, \mathbb{N}) \rightarrow$ $\operatorname{Hom}_{\mathcal{T}}\left(\mathbb{N}, \gamma_{h}(\kappa)(\mathbb{M})\right)^{\mathrm{v}}$ induced by $\operatorname{tr}^{\mathcal{T}}$.

The two descriptions are related as explained in Section 2.2. Since homological nodegeneracy of (4.12) has not been proved in general, we can view (4.20) as a conjecture for the form of the Serre functor on $\mathcal{T}$. We can test this in two particular cases for which the Serre functor is independently known.

Example 1: Minimal Landau-Ginzburg models Consider a minimal model at the conformal point. Then one can take $n=3$ and $B=\mathbb{C}\left[\phi_{1}, \phi_{2}, \phi_{3}\right]$. The weights $q_{i}$ of $\phi_{i}$ and $h$ of $W$ combine in the a regular weight vector $w:=\left(q_{1}, q_{2}, q_{3} ; h\right)$ with:

$$
\sum_{i=1}^{3} q_{i}=h+1
$$

It is well-known that such weight vectors have an ADE classification, corresponding to the type of the surface singularity defined by $W$. Namely, we have

$$
w= \begin{cases}(1, b, l+1-b ; l+1), b=1 \ldots l & \text { for } A_{l}(l \geq 1), \\ (l-2,2, l-2 ; 2(l-1)) & \text { for } D_{l}(l \geq 4), \\ (4,3,6 ; 12) & \text { for } E_{6}, \\ (6,4,9 ; 18) & \text { for } E_{7}, \\ (10,6,15 ; 30) & \text { for } E_{8} .\end{cases}
$$

and the bulk superpotential can be brought to the form:

$$
W(x, y, z)= \begin{cases}x^{l+1}+y z, & \text { for } A_{l}(l \geq 1), \\ x^{2} y+y^{l-1}+z^{2}, & \text { for } D_{l}(l \geq 4), \\ x^{3}+y^{4}+z^{2}, & \text { for } E_{6}, \\ x^{3}+x y^{3}+z^{2}, & \text { for } E_{7} \\ x^{3}+y^{5}+z^{2}, & \text { for } E_{8} .\end{cases}
$$

In all cases, the degree $h$ of $W$ coincides with the dual Coxeter number of the ADE group. Equation (4.21) reduces to the well-known formula:

$$
\hat{c}=3-2 \frac{h+1}{h}=1-\frac{2}{h} \text {. }
$$


Using this in equation (4.20), we find:

$$
S_{h}=T_{h} \tau_{h}^{-1}
$$

for all ADE groups. In [12], it was shown that $\mathcal{T}$ admits a full and strong exceptional collection, giving a triangle equivalence $\mathcal{T} \approx D^{b}\left(\bmod _{\mathbb{C} Q}\right)$, where $\mathbb{C} Q$ is the path algebra of the corresponding Dynkin quiver (whose orientation can be chosen arbitrarily). Moreover, it was shown in loc. cit that $\tau_{h}^{-1}$ agrees with the Auslander-Reiten translation [21] of $D^{b}\left(\bmod _{\mathbb{C Q}}\right)$. Thus equation (4.22) recovers the result of [28] relating the Serre and Auslender-Reiten functors of a Hom-finite Krull-Schmidt category. Also notice that (4.7) becomes the known relation between the Auslender-Reiten and translation functors of $D^{b}\left(\bmod _{\mathbb{C Q}}\right)$.

Example 2: Landau-Ginzburg models of Calabi-Yau type A Landau-Ginzburg model is of Calabi-Yau type if:

$$
\sum_{i=1}^{n} q_{i}=h
$$

In this case, in was shown in [10] that $\mathcal{T}$ is triangle equivalent with $D^{b}(X)$, where $X$ is the Calabi-Yau hypersurface $W=0$ in $W \mathbb{C P}_{q_{1} \ldots q_{n}}^{n-1}$. Equation (4.23) gives:

$$
\hat{c}=n-2=\operatorname{dim} X \text {. }
$$

Using this in (4.20), we find:

$$
S_{h}=T_{h}^{\operatorname{dim} X}
$$

which of course is the Serre functor of a Calabi-Yau variety.

\subsection{Component description}

An equivalent though less natural description of $\mathrm{DG}_{W}^{\mathrm{gr}}(B)$ can be obtained by 'passing to components' as in Section 3.1. For completeness, let us show explicitly how this recovers the formulation of $[10,12]$.

As explained in Section 3.1, a graded $B$-module of type $\left(G_{h}, \psi_{h}\right)$ can be viewed as a pair of graded $B$-modules (in our case $G_{A}=\mathbb{Z}_{2}$ ) which we denote by $M_{+}=M_{\hat{0}}$ and $M_{-}=M_{\hat{1}}$. According to the general construction, we have $M_{+}=\oplus_{\epsilon_{h}(g)=\hat{0}} M^{g}$, $M_{-}=\oplus_{\epsilon_{h}(g)=\hat{1}} M^{g}$, and the $\mathbb{Z}$-gradings on $M_{ \pm}$are specified by choosing elements $g_{ \pm} \in G$ such that $\epsilon_{h}\left(g_{+}\right)=\hat{0}$ and $\epsilon_{h}\left(g_{-}\right)=\hat{1}$. Contact with $[10,12]$ is made by choosing $g_{+}=0$ and $g_{-}=\delta_{h}$. With this choice, we have $M_{ \pm}=\oplus_{k \in \mathbb{Z}} M_{ \pm}^{k}$, where $M_{ \pm}^{k}=M^{g_{ \pm}+\psi_{h}(k)}$ are given by:

$$
M_{+}^{k}=\left\{\begin{array}{ll}
M^{(k, \hat{0})} & \text { for } h \in 2 \mathbb{N}, \\
M^{2 k} & \text { for } h \in 2 \mathbb{N}+1
\end{array} \quad, \quad M_{-}^{k}= \begin{cases}M^{\left(k+\frac{h}{2}, \hat{1}\right)} & \text { for } h \in 2 \mathbb{N} \\
M^{2 k+h} & \text { for } h \in 2 \mathbb{N}+1\end{cases}\right.
$$


Thus $M=M_{+} \oplus M_{-}$and $D$ can be decomposed as $D=\left[\begin{array}{cc}0 & F \\ G & 0\end{array}\right]$ with $F \in \operatorname{Hom}_{B}^{h}\left(M_{-}, M_{+}\right)$ and $G \in \operatorname{Hom}_{B}^{0}\left(M_{+}, M_{-}\right)$. The integrability condition $D^{2}=W$ becomes $F G=G F=$ $W$. In this language, a homogeneous morphisms $u \in \operatorname{Hom}_{\mathrm{DG}_{W}^{\mathrm{gr}}}(\mathbb{M}, \mathbb{N})$ decomposes as $u=\left[\begin{array}{ll}u_{++} & u_{-+} \\ u_{+-} & u_{--}\end{array}\right]$with $u_{\alpha \beta} \in \operatorname{Hom}_{B}\left(M_{\alpha}, N_{\beta}\right)$ and the homogeneity conditions become:

a) When $h$ is even, we have:
a1) $u \in \operatorname{Hom}_{\mathrm{DG}_{W}^{\mathrm{gr}}(B)}^{(k, \hat{)}}(\mathbb{M}, \mathbb{N})$ iff $u_{ \pm \pm} \in \operatorname{Hom}_{B}^{k}\left(M_{ \pm}, N_{ \pm}\right)$and $u_{ \pm \mp}=0$
a2) $u \in \operatorname{Hom}_{\mathrm{DG}}^{(k, \hat{1})}(\mathbb{M})(\mathbb{M}, \mathbb{N})$ iff $u_{ \pm \mp} \in \operatorname{Hom}_{B}^{k \mp \frac{h}{2}}\left(M_{ \pm}, N_{\mp}\right)$ and $u_{ \pm \pm}=0$.

b) When $h$ is odd, we have:

b1) $u \in \operatorname{Hom}_{\mathrm{DG}}^{k} \mathrm{gr}_{W}^{\mathrm{gr}(B)}(\mathbb{M}, \mathbb{N})$ with $k$ even iff $u_{ \pm \pm} \in \operatorname{Hom}_{B}^{k}\left(M_{ \pm}, N_{ \pm}\right)$and $u_{ \pm \mp}=0$

b2) $u \in \operatorname{Hom}_{\mathrm{DG}_{W}^{\mathrm{gr}}(B)}^{k}(\mathbb{M}, \mathbb{N})$ with $k$ odd iff $u_{ \pm \mp} \in \operatorname{Hom}_{B}^{\frac{k \mp h}{2}}\left(M_{ \pm}, N_{\mp}\right)$ and $u_{ \pm \pm}=0$. It is now clear that passing to the zeroth cohomology category $\mathcal{T}$ recovers the construction of $[10,12]$.

A simple case by case analysis shows that the following relations hold for all $h$ (below (1) is the usual twist functor on the category $\operatorname{gr}_{B}$ of finitely generated $\mathbb{Z}$-graded modules over $B)$ :

$$
\begin{aligned}
& \text { (1) } \tau_{h}(M, D)=\left(M\left(g_{\tau}\right), D\left(g_{\tau}\right)\right) \text { with } M\left(g_{\tau}\right)_{ \pm}=M_{ \pm}(1), D\left(g_{\tau}\right)=\left[\begin{array}{cc}
0 & F(1) \\
G(1) & 0
\end{array}\right] \text { and } \\
& \tau_{h}(u)=u\left(g_{\tau}\right)=\left[\begin{array}{ll}
u_{++} & u_{-+} \\
u_{+-} & u_{--}
\end{array}\right]=\left[\begin{array}{ll}
u_{++}(1) & u_{-+}(1) \\
u_{+-}(1) & u_{--}(1)
\end{array}\right] \\
& \text { (2) } T_{h}(M, D)=\left(M\left(g_{T}\right),-D\left(g_{T}\right)\right) \text { with } M\left(g_{T}\right)_{+}=M_{-}, M\left(g_{T}\right)_{-}=M_{+}(h), D\left(g_{T}\right)= \\
& {\left[\begin{array}{cc}
0 & G \\
F & 0
\end{array}\right] \text { and } T_{h}(u)=u\left(g_{T}\right)=\left[\begin{array}{ll}
u_{--} & u_{+-} \\
u_{-+} & u_{++}(h)
\end{array}\right] \text {. }}
\end{aligned}
$$

Passing to the zeroth cohomology category $\mathcal{T}=H^{0}\left(\mathrm{DG}_{W}^{\mathrm{gr}}(B)\right)$, it follows that $\tau_{h}$ and $T_{h}$ induce the twist and shift functors considered in [10] and [12]. In this description, we have $\operatorname{Str}_{\mathbb{M}}(u)=\operatorname{tr}_{\mathbb{M}_{+}}\left(u_{++}\right)-\operatorname{tr}_{\mathbb{M}_{-}}\left(u_{--}\right)$.

\section{Conclusion}

We discussed open 2d TFTs whose category of boundary sectors admits a grading by an arbitrary Abelian group $G$, giving a general result which translates between the physics-inspired formulation of such models and the mathematical theory of Serre functors. This provides a general method for constructing open $2 \mathrm{~d}$ TFTs with extended grading from more traditional mathematical data. When the group $G$ is sufficiently 
large, this equivalence explains how non-Calabi-Yau categories arise in the general framework of $[1,2]$.

Using this formalism, we gave a careful discussion of grading issues for the category of graded D-branes in B-type topological Landau-Ginzburg models, describing its precise relation with the triangulated category of matrix factorizations, and made a specific proposal for an equivariant Serre functor on the latter. Our result shows that the category of boundary sectors can be recovered as the graded skew category of the triangulated category of [10] with respect to the Abelian group generated by the shift and twist functors of $[10,12]$. This group depends on the parity of the bulk superpotential and recovers the correct grading on the spaces of boundary and boundary condition changing observables. We also gave a description of the dG category of graded D-branes which manifestly displays the grading by characters of the full vector-axial R-symmetry preserved by such models.

\section{A. Equivariant Serre functors.}

Let us fix a (not necessarily Abelian) group $G$. Consider a $G$-category $(\mathcal{T}, G, \gamma)$ and set $G^{*}=\operatorname{Hom}\left(G, \mathbb{C}^{*}\right)$. It is well-known that Serre functors on $\mathcal{T}$ can be described by pairs $(S, t r)$ where $S$ is an automorphism ${ }^{5}$ of $\mathcal{T}$ and $\operatorname{tr}$ is a family of linear maps $\operatorname{tr}_{a}: \operatorname{Hom}_{\mathcal{T}}(a, S(a)) \rightarrow \mathbb{C}$ define for all $a \in \mathrm{Ob} \mathcal{T}$, subject to the conditions:

$$
\operatorname{tr}_{a}(v u)=\operatorname{tr}_{b}(S(u) v) \quad \forall u \in \operatorname{Hom}_{\mathcal{T}}(a, b) \quad \forall v \in \operatorname{Hom}_{\mathcal{T}}(b, S(a))
$$

and:

$$
\operatorname{tr}_{a}(v u)=0 \quad \forall u \in \operatorname{Hom}_{\mathcal{T}}(b, S(a)) \Rightarrow u=0 .
$$

A Serre functor $(S, t r)$ on $\mathcal{T}$ is called $G$-equivariant if $S$ is $G$-equivariant and there exists a framing $\eta(g): S \circ \gamma(g) \stackrel{\sim}{\rightarrow} \gamma(g) \circ S$ of $S$ and a character $\chi \in G^{*}$ which satisfy the conditions:

$\operatorname{tr}_{\gamma(g)(a)} \gamma(g)(u)=\chi(g) \operatorname{tr}_{a}\left(\gamma(g)^{-1}\left(\eta_{a}(g)\right) \circ u\right) \forall a \in \mathrm{Ob} \mathcal{T} \forall u \in \operatorname{Hom}_{\mathcal{T}}\left(a,\left(\gamma(g)^{-1} S \gamma(g)\right)(a)\right)$

for all $g \in G$.

In the situation of Subsection 2.2, we have $G=$ Abelian, $S=G$-invariant, $\eta=\mathrm{id}=$ trivial framing and $\chi(g)=(-1)^{\epsilon(g)(\epsilon(\kappa)+1)}$ for some $\kappa \in G$.

\footnotetext{
${ }^{5}$ One can extend this to autoequivalences, but we restrict to automorphisms for simplicity.
} 


\section{B. Worldsheet analysis of R-symmetries in LG models}

Consider the B-twisted topological Landau-Ginzburg model with target $\mathbb{C}^{n}$, whose boundary coupling was given in $[5,6]$ for the simplest D-branes and constructed for arbitrary branes in $[7,8]$. In the set-up of the latter papers, the untwisted model has worldsheet bosons $\phi^{i}$ and $\phi^{\bar{i}}$ corresponding to local complex coordinates of a noncompact target Calabi-Yau manifold. It also has worldsheet fermions $\psi_{+}^{i}, \psi_{+}^{\bar{i}}$ which are sections of $K^{\frac{1}{2}} \otimes \phi^{*}\left(T^{1,0} X\right)$ and $K^{\frac{1}{2}} \otimes \phi^{*}\left(T^{0,1} X\right)$, as well as $\psi_{-}^{i}, \psi_{-}^{\bar{i}}$ which are sections of $\bar{K}^{\frac{1}{2}} \otimes \phi^{*}\left(T^{1,0} X\right)$ and $\bar{K}^{\frac{1}{2}} \otimes \phi^{*}\left(T^{0,1} X\right)$. Here $K$ and $\bar{K}$ are the canonical line bundle on the worldsheet and the bundle of $(0,1)$ forms. Performing the B-twist as in [22] replaces $\psi_{+}^{i}$ with a section $\rho_{+}^{i}$ of $K \otimes \phi^{*}\left(T^{1,0} X\right)$ and $\psi_{-}^{i}$ with a section $\rho_{-}^{i}$ of $\bar{K} \otimes \phi^{*}\left(T^{1,0} X\right)$. It also replaces $\psi_{+}^{\bar{i}}$ with a section $\chi^{\bar{i}}$ of $\phi^{*}\left(T^{0,1} X\right)$ and $\psi_{-}^{\bar{i}}$ with a section $\bar{\chi}^{\bar{i}}$ of the same bundle $\phi^{*}\left(T^{0,1} X\right)$. The later combine into the new Grassmann odd fields $\eta^{\bar{i}}=\chi^{\bar{i}}+\bar{\chi}^{\bar{i}}$ and $\theta_{i}=G_{i \bar{j}}\left(\chi^{\bar{j}}-\bar{\chi}^{\bar{j}}\right)$. Taking the bulk superpotential $W$ to be homogeneous ${ }^{6}$ of degree $h$ :

$$
W\left(\left\{e^{2 i s q_{i}} \phi_{i}\right\}\right)=e^{2 i s h} W\left(\left\{\phi_{i}\right\}\right),
$$

the model has a vector $U(1)$ R-symmetry which acts as:

$$
\begin{aligned}
& \phi^{i} \rightarrow e^{2 i s q_{i}} \phi^{i}, \quad \phi^{\bar{i}} \rightarrow e^{-2 i s q_{i}} \phi^{\bar{i}} \\
& \psi_{ \pm}^{i} \rightarrow e^{i s\left(2 q_{i}-h\right)} \psi_{ \pm}^{i}, \quad \psi_{ \pm}^{\bar{i}} \rightarrow e^{-i s\left(2 q_{i}-h\right)} \psi_{ \pm}^{\bar{i}}
\end{aligned}
$$

as well as an axial $U(1)$ R-symmetry given by:

$$
\begin{aligned}
& \phi^{i} \rightarrow \phi^{i}, \quad \phi^{\bar{i}} \rightarrow \phi^{\bar{i}}, \\
& \psi_{ \pm}^{i} \rightarrow e^{\mp 2 i t} \psi_{ \pm}^{i}, \quad \psi_{ \pm}^{\bar{i}} \rightarrow e^{ \pm 2 i t} \psi_{ \pm}^{\bar{i}}
\end{aligned}
$$

The $U(1)_{A}$ group is canonically parameterized by $e^{2 i t}$ with $t \in[0, \pi]$, while the range of $s$ depends on the parity of $h$. For even $h$, we take $s \in[0, \pi]$, while for odd $h$ we take $s \in[0,2 \pi]$. This insures that the action of the vector R-symmetry group is a proper representation. Notice that for odd $h$, the $U(1)_{V}$ group is the double cover of the circle $\left\{e^{2 i s} \mid s \in[0, \pi]\right\}$; of course, the double cover is again a circle group. These actions translate as follows for the Grassmann-odd fields of the twisted theory:

$$
\rho^{i} \rightarrow e^{i s\left(2 q_{i}-h\right)} \rho^{i} \quad, \quad \eta^{\bar{i}} \rightarrow e^{-i s\left(2 q_{i}-h\right)} \eta^{\bar{i}}, \theta_{i} \rightarrow e^{-i s\left(2 q_{i}-h\right)} \theta^{\bar{i}}
$$

for $U(1)_{V}$ and:

$$
\rho_{ \pm}^{i} \rightarrow e^{\mp 2 i t} \rho_{ \pm}^{i}, \quad, \quad \chi^{\bar{i}} \rightarrow e^{+2 i t} \chi^{\bar{i}}, \quad \bar{\chi}_{i} \rightarrow e^{-2 i t} \bar{\chi}^{\bar{i}}
$$

\footnotetext{
${ }^{6}$ Due to the superspace formulation, it is traditional to take $W$ to formally have degree 2 by using the 'fractional charges' $\tilde{q}_{i}:=2 q_{i} / h$ instead of $q_{i}$. Here we prefer to work with integral charges since they are directly related to the characters of $U(1)$.
} 
for $U(1)_{A}$. As explained in [7, 8], a general topological D-brane of this model is described by a superbundle $E=E_{+} \oplus E_{-}$over the target space, together with a superconnection $\mathcal{A}$ whose super-curvature can be taken to satisfy $\mathcal{F}^{(0, \leq 2)}=W$. The boundary coupling is given by the super-Wilson loops:

$$
\mathcal{U}:=\operatorname{Str} P e^{-\oint_{C} d \tau \mathcal{M}}
$$

where $C$ is a circle boundary of the worldsheet. The quantity $\mathcal{M}$ is given by:

$$
\mathcal{M}=\left[\begin{array}{cc}
\hat{\mathcal{A}}^{(+)}+\frac{i}{2}\left(F F^{\dagger}+G^{\dagger} G\right) & \frac{1}{2} \rho_{0}^{i} \nabla_{i} F+\frac{i}{2} \eta^{\bar{i}} \nabla_{\bar{i}} G^{\dagger} \\
\frac{1}{2} \rho_{0}^{i} \nabla_{i} G+\frac{i}{2} \eta^{\bar{i}} \nabla_{\bar{i}} F^{\dagger} & \hat{\mathcal{A}}^{(-)}+\frac{i}{2}\left(F^{\dagger} F+G G^{\dagger}\right)
\end{array}\right]
$$

where $\rho_{0}^{i} d \tau_{a}$ is the pull-back of $\rho^{i}$ to $C_{a}$ and:

$$
\hat{\mathcal{A}}^{( \pm)}:=A_{\bar{i}}^{( \pm)} \dot{\phi}^{\bar{i}}+\frac{1}{2} \eta^{\bar{i}} F_{\bar{i} j}^{( \pm)} \rho_{0}^{j}
$$

are connections on the bundles $\mathcal{E}_{ \pm}$obtained by pulling back $E_{ \pm}$to the boundary. The dot stands for the derivative $\frac{d}{d \tau}$. One has $\mathcal{M}=\hat{\mathcal{A}}+\Delta+K$, where:

$$
\begin{gathered}
\Delta:=\frac{1}{2} \rho_{0}^{i} \partial_{i} D \\
K:=\frac{i}{2}\left(\eta^{\bar{i}} \nabla_{\bar{i}} D^{\dagger}+\left[D, D^{\dagger}\right]_{+}\right)
\end{gathered}
$$

and:

$$
\hat{\mathcal{A}}=\dot{\phi}^{\bar{i}} A_{\bar{i}}+\frac{1}{2} F_{\bar{i} j} \eta^{\bar{i}} \rho_{0}^{j}
$$

Here $A=A_{i} d \phi^{i}+A_{\bar{i}} d \phi^{\bar{i}}=A^{(+)}+A^{(-)}$is the direct sum connection on $\operatorname{End}(E)$ induced by the 1 -form part of $\mathcal{A}$, while $D=\left[\begin{array}{cc}0 & F \\ G & 0\end{array}\right] \in \operatorname{End}^{\text {odd }}(E)$ is the zero-form part of $\mathcal{A}$, which satisfies $D^{2}=W$ and plays the role of topological tachyon condensate.

Consider the coordinate ring $B=\mathbb{C}\left[\phi_{1} \ldots \phi_{n}\right]$ of the target space $\mathbb{C}^{n}$. Passing to holomorphic sections of $E$, one finds $[7,8]$ that boundary conditions correspond to pairs $\mathbb{M}=(M, D)$ where $M$ is a free finitely-generated $B$-supermodule and $D \in \operatorname{End}_{B}^{\text {odd }}(M)$ satisfies the constraint $D^{2}=W$. We have a $\mathbb{Z}_{2}$-graded dG category $\mathrm{DG}_{W}(B)$ whose objects are pairs of this form and whose morphisms are $\operatorname{Hom}_{\mathbb{D G}_{W}(B)}(\mathbb{M}, \mathbb{N}):=\operatorname{Hom}_{B}(M, N)$, endowed with the differential $d_{\mathbb{M}, \mathbb{N}}(u)=D_{N} \circ$ $u-(-1)^{|u|} u \circ D_{M}$, where $|u| \in \mathbb{Z}_{2}$ denotes the $\mathbb{Z}_{2}$-degree of $u$. This is the dG category of all topological D-branes. The space of boundary observables reduces to $H_{d_{\mathbb{M N}}}\left(\operatorname{Hom}_{B}(M, N)\right)$. 
The axial R-symmetry is broken by the bulk superpotential to the subgroup $\Gamma_{A} \approx$ $\mathbb{Z}_{2}$ whose generator corresponds to $t=\frac{\pi}{2}$ in (B.4), giving the residual $\mathbb{Z}_{2}$ action:

$$
\rho^{i} \rightarrow-\rho^{i}, \eta^{\bar{i}} \rightarrow-\eta^{\bar{i}}, \quad \theta_{i} \rightarrow-\theta^{\bar{i}} .
$$

One finds that the boundary coupling is invariant if one takes the tachyon condensate to transform as:

$$
D \rightarrow U_{A} D U_{A}^{-1}=-D,
$$

where $U_{A}=\left[\begin{array}{cc}1 & 0 \\ 0 & -1\end{array}\right]$ satisfies $U_{A}^{2}=1$. This shows that we must take the residual axial R-symmetry to act on $\operatorname{Hom}_{B}(M, N)$ as $u \rightarrow U_{A} u U_{A}^{-1}$, i.e.:

$$
u \rightarrow(-1)^{|u|} u \quad \forall \text { homogeneous } u \in \operatorname{Hom}_{B}(M, N) .
$$

Hence the $\mathbb{Z}_{2}$-grading of $\mathrm{DG}_{W}(B)$ is induced by this residual symmetry.

The connections $A^{( \pm)}$are $U(1)_{V}$-invariant, which amounts to the component transformation laws $A_{i}^{( \pm)}\left(\left\{e^{2 i s q_{i}} \phi_{i}\right\}\right)=e^{-2 i s q_{i}} A_{i}^{( \pm)}\left(\left\{\phi_{i}\right\}\right)$ and $A_{\bar{i}}^{( \pm)}\left(\left\{e^{2 i s q_{i}} \phi_{i}\right\}\right)=e^{+2 i s q_{i}} A_{\bar{i}}^{( \pm)}\left(\left\{\phi_{i}\right\}\right)$. It is easy to check that the boundary coupling is $U(1)_{V}$-invariant provided that $F$ and $G$ satisfy:

$$
F\left(\left\{e^{2 i s q_{i}} \phi_{i}\right\}\right)=e^{2 i s h} F\left(\left\{\phi_{i}\right\}\right), \quad G\left(\left\{e^{2 i s q_{i}} \phi_{i}\right\}\right)=G\left(\left\{\phi_{i}\right\}\right) .
$$

Indeed, this corresponds to $U_{V}(s) D\left(\left\{e^{2 i s q_{i}} \phi_{i}\right\}\right) U_{V}(s)^{-1}=e^{i s h} D\left(\left\{\phi_{i}\right\}\right)$, where $U_{V}(s):=$ $\left[\begin{array}{cc}1 & 0 \\ 0 & e^{i s h}\end{array}\right]$ gives a representation of $U(1)_{V}$. Then (B.6) satisfies $\mathcal{M}\left(\left\{e^{2 i s q_{i}} \phi_{i}\right\}\right)=U_{V}(s)^{-1} \mathcal{M}\left(\left\{\phi_{i}\right\}\right) U_{V}(s)$ so (B.5) is invariant. We have $\Gamma_{V} \approx U(1)$ and $\Gamma_{V}^{*} \approx U(1)^{*} \approx \mathbb{Z}$.

To take the vector R-symmetry into account, we grade the ring $B$ by associating weights $q_{i}$ to $\phi_{i}$. The analysis of [9] shows that $U(1)_{V}$-invariant boundary conditions correspond to those pairs $\mathbb{M}=(M, D) \in \mathrm{ObDG}_{W}$ for which $M_{ \pm}$are free graded $B$-modules and $D=\left[\begin{array}{cc}0 & F \\ G & 0\end{array}\right]$ is such that $F$ and $G$ are homogeneous of degrees $h$ and 0 respectively. We will refer to this grading as the 'vector grading'. The dG category of 'graded branes' is the full subcategory $\mathrm{DG}_{W}^{\mathrm{gr}}(B)$ of $\mathrm{DG}_{W}(B)$ consisting of such pairs; this is the $\mathrm{dG}$ category of those topological D-branes whose boundary conditions preserve the full $U(1)_{V}$ symmetry. For any objects $\mathbb{M}$ and $\mathbb{N}$ of $D G_{W}^{g r}$, we have an action of $U(1)_{V}$ on $\operatorname{Hom}_{B}(\mathbb{M}, \mathbb{N})$ given by:

$$
u \rightarrow U_{V}(s) u\left(\left\{e^{2 i s q_{i}} \phi_{i}\right\}\right) U_{V}(s)^{-1},
$$

as well as the action of the axial $\mathbb{Z}_{2}$ symmetry:

$$
u \rightarrow U_{A} u U_{A}^{-1} .
$$


Since $\left[U_{A}, U_{V}(s)\right]=0$, these combine into an action of the group $\Gamma=\Gamma_{V} \times \Gamma_{A} \approx$ $U(1) \times \mathbb{Z}_{2}$.

The morphism $u \in \operatorname{Hom}_{\mathrm{DG}_{W}^{\mathrm{gr}}(B)}(\mathbb{M}, \mathbb{N})$ is 'vector homogeneous' of degree $q \in \frac{1}{2} \mathbb{Z}$ if it satisfies:

$$
U_{V}(s) u\left(\left\{e^{2 i s q_{i}} \phi_{i}\right\}\right) U_{V}(s)^{-1}=e^{2 i s q} u\left(\left\{\phi_{i}\right\}\right) .
$$

With this definition, the condensate $D$ of every object $\mathbb{M}=(M, D)$ of $\operatorname{DG}_{W}^{\mathrm{gr}}(B)$ is homogeneous of vector degree $\frac{h}{2}$. Writing $u=\left[\begin{array}{l}u_{++} u_{-+} \\ u_{+-} u_{--}\end{array}\right]$with $u_{\alpha \beta} \in \operatorname{Hom}_{B}\left(M_{\alpha}, N_{\beta}\right)$, this condition amounts to:

$$
\operatorname{deg} u_{++}=\operatorname{deg} u_{--}=q, \operatorname{deg} u_{-+}=q+\frac{h}{2}, \quad \operatorname{deg} u_{+-}=q-\frac{h}{2} .
$$

Since $u_{\alpha \beta}$ must have integral degrees, this requires $q \in \mathbb{Z}$ for $u \in \operatorname{Hom}_{\mathrm{DG}_{W}^{\mathrm{gr}}(B)}^{\hat{0}}(\mathbb{M}, \mathbb{N})$ and $q \in \mathbb{Z}+\frac{h}{2}$ for $u \in \operatorname{Hom}_{\mathrm{DG}_{W}^{\mathrm{gr}}(B)}^{\hat{\mathrm{g}}}(\mathbb{M}, \mathbb{N})$. We find the decompositions:

$$
\begin{aligned}
& \operatorname{Hom}_{\mathrm{DG}}^{\hat{0}} \mathrm{gr}_{W}^{\mathrm{gr}(B)}(\mathbb{M}, \mathbb{N})=\oplus_{q \in \mathbb{Z}} \operatorname{Hom}_{\mathrm{DG}_{W}^{\mathrm{gr}}(B)}^{[q, \hat{g}]}(\mathbb{M}, \mathbb{N}) \\
& \operatorname{Hom}_{\mathrm{DG}}^{\hat{1}} \mathrm{gr}_{W}^{\mathrm{gr}(B)}(\mathbb{M}, \mathbb{N})=\oplus_{q \in \mathbb{Z}+\frac{h}{2}} \operatorname{Hom}_{\mathrm{DG}_{W}^{\mathrm{gr}(B)}}^{[q, \hat{\mathrm{g}}]}(\mathbb{M}, \mathbb{N})
\end{aligned}
$$

We have $D_{\mathbb{M}} \in \operatorname{Hom}_{\mathrm{DG}_{W}^{\mathrm{gr}}(B)}^{\left[\frac{h}{2}, \hat{1}\right]}(\mathbb{M}, \mathbb{M})$, so the differential $d_{\mathbb{M}, \mathbb{N}}$ satisfies:

$$
d_{\mathbb{M}, \mathbb{N}}\left(\operatorname{Hom}_{\mathrm{DG}_{W}^{\mathrm{gr}}(B)}^{[q, \alpha]}(\mathbb{M}, \mathbb{N})\right) \subset \operatorname{Hom}_{\mathrm{DG}_{W}^{\mathrm{gr}}(B)}^{\left[q+\frac{h}{2}, \alpha+\hat{1}\right]}(\mathbb{M}, \mathbb{N})
$$

When $h$ is even, we have $\mathbb{Z}+\frac{h}{2}=\mathbb{Z}$ and the decompositions above give a $\mathbb{Z} \times \mathbb{Z}_{2}$-grading:

$$
\operatorname{Hom}_{\mathrm{DG}_{W}^{\mathrm{gr}}(B)}(\mathbb{M}, \mathbb{N})=\oplus_{q \in \mathbb{Z}, \alpha \in \mathbb{Z}_{2}} \operatorname{Hom}_{\mathrm{DG}_{W}^{\mathrm{gr}}(B)}^{[q, \hat{\mathrm{g}}]}(\mathbb{M}, \mathbb{N})
$$

When $h$ is odd, we have $\mathbb{Z}+\frac{h}{2}=\left(\frac{1}{2} \mathbb{Z}\right) \backslash \mathbb{Z}$ and we find a $\frac{1}{2} \mathbb{Z}$-grading:

$$
\operatorname{Hom}_{\mathrm{DG}_{W}^{\mathrm{gr}}(B)}(\mathbb{M}, \mathbb{N})=\oplus_{q \in \frac{1}{2} \mathbb{Z}} \operatorname{Hom}_{\mathrm{DG}_{W}^{\mathrm{gr}}(B)}^{[q]}(\mathbb{M}, \mathbb{N})
$$

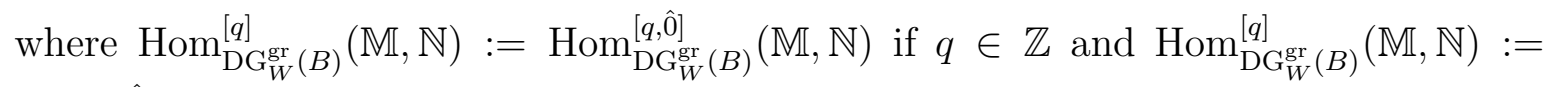
$\operatorname{Hom}_{\mathrm{DG}_{W}^{\mathrm{gr}}(B)}^{[q, \hat{1}]}(\mathbb{M}, \mathbb{N})$ if $q \in\left(\frac{1}{2} \mathbb{Z}\right) \backslash \mathbb{Z}$.

It is easy to see that both of these decompositions are compatible with the composition of morphisms. Equation (B.9) shows that $d_{\mathbb{M N}}$ are homogeneous of bidegree $\left(\frac{h}{2}, \hat{1}\right) \in \mathbb{Z} \times \mathbb{Z}_{2}$ when $h$ is even, and of degree $\frac{h}{2} \in \mathbb{Z}$ when $h$ is odd. Setting:

$$
G_{h}= \begin{cases}\mathbb{Z} \times \mathbb{Z}_{2} & \text { for } h \in 2 \mathbb{N} \\ \mathbb{Z} & \text { for } h \in 2 \mathbb{N}+1\end{cases}
$$


as in (4.1), we redefine the grading for odd $h$ so that all degrees become integral. Thus we set $\operatorname{Hom}_{\mathrm{DG}_{W}^{\mathrm{gr}}(B)}(\mathbb{M}, \mathbb{N})=\oplus_{g \in G_{h}} \operatorname{Hom}_{\mathrm{DG}}^{g} \mathrm{gr}_{W}^{\mathrm{gr}}(B)(\mathbb{M}, \mathbb{N})$, where

$$
\operatorname{Hom}_{\mathrm{DG}_{W}^{\mathrm{gr}}(B)}^{g}(\mathbb{M}, \mathbb{N})=\left\{\begin{array}{l}
\operatorname{Hom}_{\mathrm{DG}_{W}^{\mathrm{gr}}(B)}^{[k, \alpha]}(\mathbb{M}, \mathbb{N}) \text { for } h \in 2 \mathbb{N} \text { and } g=(k, \alpha) \in \mathbb{Z} \times \mathbb{Z}_{2} \\
\operatorname{Hom}_{\mathrm{DG}_{W}^{\mathrm{gr}}(B)}^{k k / 2]}(\mathbb{M}, \mathbb{N}) \text { for } h \in 2 \mathbb{N}+1 \text { and } g=k \in \mathbb{Z}
\end{array}\right.
$$

With this convention, $\mathrm{DG}_{W}^{\mathrm{gr}}(B)$ is a $G_{h}$-graded category. We let $\operatorname{deg} u \in G_{h}$ denote the $G_{h}$-degree of morphisms, which is given by:

$$
\operatorname{deg} u= \begin{cases}(q, \alpha) \in \mathbb{Z} \times \mathbb{Z}_{2} & \text { for } h \in 2 \mathbb{N} \\ 2 q \in \mathbb{Z} & \text { for } h \in 2 \mathbb{N}+1\end{cases}
$$

Notice that the rescaled vector R-charge of $u$ is recovered as $\phi_{h}(\operatorname{deg} u)$, where $\phi_{h}: G \rightarrow$ $\mathbb{Z}$ is the group morphism given by:

$$
\left\{\begin{array}{ll}
\phi_{h}(k, \alpha)=k & \text { for } h \in 2 \mathbb{N} \\
\phi_{h}(k)=k & \text { for } h \in 2 \mathbb{N}+1
\end{array} .\right.
$$

The true vector R-charge (which is valued in $\frac{1}{2} \mathbb{Z}$ ) is given by:

$$
q(u)= \begin{cases}\phi_{h}(\operatorname{deg} u) & \text { for } h \in 2 \mathbb{N} \\ \frac{1}{2} \phi_{h}(\operatorname{deg} u) & \text { for } h \in 2 \mathbb{N}+1\end{cases}
$$

On the other hand, the axial charge is recovered as $\epsilon_{h}(\operatorname{deg} u)$, where $\epsilon_{h}: G_{h} \rightarrow \mathbb{Z}_{2}$ is the group morphism given in (4.4).

The above can also be described as follows. We have a decomposition:

$$
M=\oplus_{q \in \mathbb{Z}} M^{[q, \hat{0}]} \oplus \oplus_{q \in \mathbb{Z}+\frac{h}{2}} M^{[q, \hat{1}]},
$$

with $M^{[q, \hat{0}]}=M_{+}^{q}$ and $M^{[q, \hat{1}]}=M_{-}^{q-\frac{h}{2}}$, where $M_{ \pm}=\oplus_{k \in \mathbb{Z}} M_{ \pm}^{k}$ are the decompositions of $M_{ \pm}$as graded $B$-modules. It is clear that the components satisfy $M^{[q, \alpha]} B^{k} \subset M^{[q+k, \alpha]}$.

For even $h$, equation (B.11) gives a $\mathbb{Z} \times \mathbb{Z}_{2}$ grading $M=\oplus_{q \in \mathbb{Z}, \alpha \in \mathbb{Z}_{2}} M^{[q, \alpha]}$, while for odd $h$ it gives a $\frac{1}{2} \mathbb{Z}$-grading $M=\oplus_{q \in \frac{1}{2} \mathbb{Z}} M^{[q]}$, where $M^{[q]}:=M^{[q, \hat{0}]}$ for $q \in \mathbb{Z}$ and $M^{[q]}:=M^{[q, \hat{1}]}$ for $q \in \mathbb{Z}+\frac{1}{2}$. Rescaling $q$ for odd $h$ such that all charges become

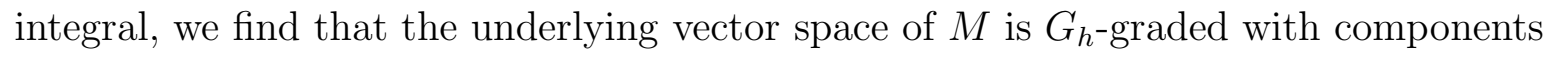
$M^{(q, \alpha)}=M^{[q, \alpha]}$ for $h$ even, and $M^{q}:=M^{[q / 2]}$ for $h$ odd. This rescaled grading satisfies $M^{g} B^{k} \subset M^{g+\psi_{h}(k)}$, where the morphism $\psi_{h}: \mathbb{Z} \rightarrow G$ is given in (4.3). Hence the objects of $\mathrm{DG}_{W}^{\mathrm{gr}}(B)$ are integrable modules over the curved differential graded algebra $(B, 0, W)$, as discussed in Section 4. The grading (B.10) on $\mathrm{DG}_{W}^{\mathrm{gr}}(B)$ is naturally induced by these $G_{h}$-gradings on objects. 
Finally, we notice that the difference made by the parity of $h$ can also be seen directly. The group $\Gamma=\Gamma_{V} \times \Gamma_{A}=U(1) \times \mathbb{Z}_{2}$ acts on $\operatorname{Hom}_{\mathrm{DG}_{W}^{\mathrm{gr}}(B)}(\mathbb{M}, \mathbb{N})$ by the product of the actions (B.7) and (B.8). This combined action is faithful for generic $\mathbb{M}, \mathbb{N}$ when $h$ is even, but has a $\mathbb{Z}_{2}$ kernel when $h$ is odd (in the the latter case we have $\left.U_{V}(\pi)=U_{A}\right)$. Hence the 'effective' symmetry group equals $U(1) \times \mathbb{Z}_{2}$ when $h$ is even and $\left[U(1) \times \mathbb{Z}_{2}\right] / \mathbb{Z}_{2} \approx U(1)$ when $h$ is odd. As a consequence, its group of characters equals $\mathbb{Z} \times \mathbb{Z}_{2}$ or $\mathbb{Z}$ respectively. Of course, this is the group $G_{h}$ considered above.

\section{References}

[1] C. I. Lazaroiu, "On the structure of open-closed topological field theory in two dimensions," Nucl. Phys. B 603 (2001) 497 [arXiv:hep-th/0010269].

[2] G. W. Moore, "Some comments on branes, G-flux, and K-theory," Int. J. Mod. Phys. A 16 (2001) 936 [arXiv:hep-th/0012007].

[3] A. I. Bondal, M. M. Kapranov, "Representable functors, Serre functors and mutations", Math. USSR Izv. 1990, 35 (3), 519-541.

[4] C. Cibils, E. N. Marcos, "Skew category, Galois covering and smash product of a $k$ category", math.RA/0312214.

[5] A. Kapustin, Yi Li, "D-branes in Topological Minimal Models: the Landau-Ginzburg Approach", JHEP 0407 (2004) 045 [hep-th/0306001].

[6] I. Brunner, M. Herbst, W. Lerche, B. Scheuner, "Landau-Ginzburg Realization of Open String TFT", hep-th/0305133.

[7] C.I. Lazaroiu, "On the boundary coupling of topological Landau-Ginzburg models", JHEP 0505 (2005) 037 [hep-th/0404184].

[8] M. Herbst, C. I. Lazaroiu, "Localization and traces in open-closed topological LandauGinzburg models", JHEP 0505 (2005) 044 [hep-th/0404184.]

[9] J. Walcher, "Stability of Landau-Ginzburg branes", J. Math. Phys. 46 (2005) 082305, [hep-th/0412274].

[10] D. Orlov, "Derived categories of coherent sheaves and triangulated categories of singularities", math.AG/0503632.

[11] A. Takahashi, "Matrix Factorizations and Representations of Quivers I", math.AG/0506347.

[12] H. Kajiura, K. Saito, A. Takahashi, "Matrix Factorizations and Representations of Quivers II: type ADE case", math.AG/0511155. 
[13] C. I. Lazaroiu, "Generalized complexes and string field theory," JHEP 0106 (2001) 052 [arXiv:hep-th/0102122].

[14] C. I. Lazaroiu, "Unitarity, D-brane dynamics and D-brane categories," JHEP 0112 (2001) 031 [arXiv:hep-th/0102183].

[15] C. I. Lazaroiu, "Graded Lagrangians, exotic topological D-branes and enhanced triangulated categories", JHEP 0106, 064 (2001), [arXiv:hep-th/0105063].

[16] D.-E. Diaconescu, "Enhanced D-Brane Categories from String Field Theory", JHEP 0106 (2001) [hep-th/0104200].

[17] C. I. Lazaroiu, R. Roiban, "Holomorphic potentials for graded D-branes," JHEP 0202 (2002) 038 [arXiv:hep-th/0110288].

[18] C. I. Lazaroiu, R. Roiban and D. Vaman, "Graded Chern-Simons field theory and graded topological D-branes," JHEP 0204 (2002)023 [arXiv:hep-th/0107063].

[19] C. I. Lazaroiu, R. Roiban, "Gauge-fixing, semiclassical approximation and potentials for graded Chern-Simons theories," JHEP 0203 (2002) 022 [arXiv:hep-th/0112029].

[20] J. Block, "Duality and equivalence of module categories in noncommutative geometry I", math.QA/0509284.

[21] D. Happel, "Triangulated categories in the representation theory of finite-dimensional algebras", Cambridge University Press, Cambridge, 1988.

[22] E. Witten, "Mirror manifolds and topological field theory", Essays on mirror manifolds, 120-158, Internat. Press, Hong Kong, 1992 [hep-th/9112056].

[23] C. I. Lazaroiu, "Generating the superpotential on a D-brane category: I", hepth/0610120.

[24] D. Orlov, "Triangulated categories of singularities and D-branes in Landau-Ginzburg models", math.AG/0302304.

[25] D. Orlov, "Triangulated categories of singularities and equivalences between LandauGinzburg models", math.AG/0503630.

[26] K. Fukaya, Y.-G. Oh, H. Ohta, K. Ono, "Lagrangian intersection Floer theory - anomaly and obstruction", preprint available at http : //www.kusm.kyoto u.ac.jp/ fukaya/fukaya.html.

[27] B. Keller, "On triangulated orbit categories", Documenta Math. 10 (2005), 551-581 [math.RT/0503240]. 
[28] I. Reiten, M. Van den Bergh, "Noetherian hereditary categories satisfying Serre duality", J. Amer. Math. Soc. 15 (2002) 295-366 [math.RT/9911242]

[29] R. Bocklandt, "Graded Calabi Yau Algebras of dimension 3", math.RA/0603558 